\title{
CONVERGENCE, UNIQUENESS, AND SUMMABILITY OF MULTIPLE TRIGONOMETRIC SERIES
}

\author{
BY \\ J. MARSHALL ASH( $\left.{ }^{1}\right)$ AND GRANT V. WELLAND $\left({ }^{2}\right)$
}

\begin{abstract}
In this paper our primary interest is in developing further insight into convergence properties of multiple trigonometric series, with emphasis on the problem of uniqueness of trigonometric series. Let $E$ be a subset of positive (Lebesgue) measure of the $k$ dimensional torus. The principal result is that the convergence of a trigonometric series on $E$ forces the boundedness of the partial sums almost everywhere on $E$ where the system of partial sums is the one associated with the system of all rectangles situated symmetrically about the origin in the lattice plane with sides parallel to the axes. If $E$ has a countable complement, then the partial sums are bounded at every point of $E$. This result implies a uniqueness theorem for double trigonometric series, namely, that if a double trigonometric series converges unrestrictedly rectangularly to zero everywhere, then all the coefficients are zero. Although uniqueness is still conjectural for dimensions greater than two, we obtain partial results and indicate possible lines of attack for this problem.

We carry out an extensive comparison of various modes of convergence (e.g., square, triangular, spherical, etc.). A number of examples of pathological double trigonometric series are displayed, both to accomplish this comparison and to indicate the "best possible" nature of some of the results on the growth of partial sums.

We obtain some compatibility relationships for summability methods and finally we present a result involving the $(C, \alpha, 0)$ summability of multiple Fourier series.
\end{abstract}

Introduction. The main interest of this paper will be the theory of multiple trigonometric series. Multiple Fourier series (the most important type of multiple trigonometric series) will be discussed only in connection with the theory of uniqueness and again in the last chapter. For the definitions of any unfamiliar terms used in the introduction the reader is referred to $\S I$.

One of the main difficulties in multiple series arises in connection with the usual consistency theorems for summation methods. In order to maintain the validity of the typical theorem "convergence implies summability," even in the case of Poisson summation one has to have the added condition that all partial sums be bounded. If one attempts to restrict himself to regular methods of forming the partial sums, it is easy to construct examples where this condition fails. However, by introducing unrestricted rectangular partial sums, convergence of a multiple trigonometric

Received by the editors January 22, 1971.

AMS 1970 subject classifications. Primary 42A92, 42A48, 42A20, 42A24, 40B05; Secondary 40G10, 40A05, 40D15.

Key words and phrases. Square summable, unrestricted rectangularly convergent, spherically Abel summable, $(C, 0, \beta)$ summable, uniqueness, coefficient growth, Riemann summable.

(1) Research partially supported by NSF Grant No. GP-14986.

${ }^{(2)}$ Research partially supported by NSF Grant GP-9!23.

Copyright (C) 1972, American Mathematical Society 
series on a set of positive measure implies the pointwise boundedness of the partial sums almost everywhere in the given set. Hence, one achieves a wide range of consistency theorems on an almost everywhere basis.

The technique of obtaining the boundedness of the partial sums has as its origin a work of P. J. Cohen [2], in which the author obtains an estimate for the rate of increase of coefficients of a multiple trigonometric series convergent almost everywhere by a regular method. By applying this technique (it is described and applied in $\S I$ I) in the case of unrestricted rectangular convergence in a set of positive measure, one is able to even conclude that the coefficients are bounded (Theorem 2.2). Lemma 2.1, part (b) of Lemma 2.2, and the first statement in Theorem 2.1 were proved by P. J. Cohen in [2]. We reproduce the proofs here since [2] is not easily available and since the other applications show Cohen's technique to be more powerful and useful than had previously been apparent.

Another difficulty in multiple series is the diversity of possible partial sums. As is pointed out in §III, this diversity introduces problems at the most fundamental level. Perhaps the most "natural" methods of forming partial sums are by circles, squares, rectangles, and diamonds (corresponding to diamonds is triangular convergence). Several examples are given in §III to show the basic incompatibilities between these methods. See Figure 3 for a summary of the situation. This incompatibility makes somewhat surprising the fact that convergence (三unrestricted rectangular convergence) everywhere implies spherical Abel summability everywhere (Theorem 3.1).

In $\S \mathrm{IV}$, the fact from §II that everywhere convergence controls the growth of the coefficients and the fact from §III that convergence implies spherical Abel summability are combined with a uniqueness theorem of V. Shapiro [16] to obtain uniqueness for double trigonometric series that converge in a set which excludes at most one point. The question of uniqueness under the hypothesis of everywhere convergence is still open in dimensions greater than two. We give three other possible approaches with partial results. One result (Theorem 4.3) is that uniqueness holds for multiple trigonometric series of power series type that converge everywhere.

Finally in $\S \mathrm{V}$ some recently proved results in one dimensional Fourier series [1], [9] are used to prove $(C, \alpha, 0)$ summability for certain double Fourier series $(\alpha>0)$. This result complements certain recent results of C. Fefferman [6], [7], P. Sjolin [19], and N. Tevzadze [20] concerning the convergence and divergence of multiple Fourier series.

For further references the reader is referred to the excellent bibliography in [18].

I. Definitions. In this paper, we will be considering various aspects of pointwise convergence of a multiple trigonometric series

$$
T(x)=\sum a_{m} e^{i m \cdot x}
$$


We use $\boldsymbol{m}=\left(m_{1}, m_{2}, \ldots, m_{k}\right)$ where the $m_{i}$ are integers and $\boldsymbol{x}=\left(x_{1}, x_{2}, \ldots, x_{k}\right)$ $\in T^{k}$. We will understand $T^{k}=[0,2 \pi) \times[0,2 \pi) \times \cdots \times[0,2 \pi)$ to be $E^{k}$ with two vectors $\boldsymbol{x}$ and $\boldsymbol{y}$ identified whenever $x_{i} \equiv y_{i}(\bmod 2 \pi), i=1, \ldots, k$. Also, $|\boldsymbol{x}-\boldsymbol{y}|$ $=\min \left\{\left[\sum_{i=1}^{k}\left(x_{i}^{*}-y_{i}^{*}\right)^{2}\right]^{1 / 2}: x^{*}\right.$ is equivalent to $\boldsymbol{x}$ and $\boldsymbol{y}^{*}$ is equivalent to $\left.\boldsymbol{y}\right\}$. For $z \in C,|z|$ means the usual modulus of a complex number. We set $\boldsymbol{m} \cdot \boldsymbol{x}=m_{1} x_{1}$ $+\cdots+m_{k} x_{k},|\boldsymbol{m}|=\left(m_{1}^{2}+\cdots+m_{k}^{2}\right)^{1 / 2},\|\boldsymbol{m}\|=\min _{i}\left\{\left|m_{i}\right|\right\}$, and $\|\boldsymbol{m}\|=\max _{i}\left\{\left|m_{i}\right|\right\}$. The relation $\boldsymbol{m} \geqq \boldsymbol{n}$ will mean $m_{i} \geqq n_{i}, i=1, \ldots, k$. Let $\Omega^{k}$ be the set of all $k$-tuples $p=\left(p_{1}, \cdots, p_{k}\right)$ where $p_{i}=0$ or 1 for $i=1, \ldots, k$. If $p \in \Omega^{k}$, we set

$$
\left(\frac{\partial}{\partial x}\right)^{p} f=\frac{\partial}{\partial x_{i_{1}}} \frac{\partial}{\partial x_{i_{2}}} \cdots \frac{\partial}{\partial x_{i_{r}}} f \text { and }(d x)^{p}=d x_{i_{1}} d x_{i_{2}} \cdots d x_{i_{r}}
$$

where $p_{i_{s}}=1, s=1, \ldots, r$ and $p_{j}=0$ otherwise. When $k=2$, we will take $\boldsymbol{m}=(m, n)$, $\boldsymbol{x}=(x, y),|\boldsymbol{m}|=\left(m^{2}+n^{2}\right)^{1 / 2}$ and so forth in order to simplify the notation.

There are many interpretations of the statement " $T$ converges at $x$." A method of convergence is described by a sequence $\left\{E_{n}\right\}_{n=0,1,2, \ldots}$ of finite sets of $k$-tuples of integers such that each $E_{n}$ is contained in $E_{n+j}$ for all sufficiently large $j$ and the union of all the $E_{n}$ consists of all $k$-tuples of integers. The method is symmetric if $\boldsymbol{m} \in E_{n}$ implies that so are the other $k$-tuples $\boldsymbol{m}^{\prime}$ satisfying $\left|m_{i}^{\prime}\right|=\left|m_{i}\right|, i=1, \ldots, k$. In this paper only symmetric methods will be considered.

A method of convergence is regular if and only if there is a constant $K$ such that for every lattice point $\boldsymbol{m}=\left(m_{1}, \ldots, m_{k}\right)$ there exists $n_{0}$ such that $\boldsymbol{m}$ belongs to $E_{n_{0}}$ and such that for each lattice point $\boldsymbol{l}$ in $E_{n_{0}}$ we have $\|\boldsymbol{l}\| \leqq K\|\boldsymbol{m}\|$. We call the least such $K$ the eccentricity of the method. In other words, the $E_{n}$ 's are not too eccentrically shaped. This definition is due to Paul J. Cohen [2, p. 39].

Some examples of regular methods of convergence are spherical convergence or circular convergence if $k=2\left(E_{n}=\{\boldsymbol{m}|| \boldsymbol{m} \mid \leqq n\}\right)$, triangular convergence $\left(E_{n}=\left\{\boldsymbol{m}\left|\sum_{i=1}^{k}\right| m_{i} \mid \leqq n\right\}\right)$, square convergence $\left(E_{n}=\{\boldsymbol{m}|\|\boldsymbol{m}\|| \leqq n)\right.$, and restricted rectangular convergence. $T$ is said to converge restrictedly rectangularly if $T$ converges to the same value for every sequence $\left\{E_{n}\right\}$ satisfying the following conditions:

(i) each $E_{n}$ is a rectangle symmetric about the origin with sides parallel to the axes;

(ii) if $p_{n}$ denotes the minimum dimension of $E_{n}$ and if $q_{n}$ denotes its maximum dimension, then $q_{n} / p_{n}$ is uniformly bounded.

The minimum bound will be called the eccentricity of the sequence $\left\{E_{n}\right\}$.

If condition (ii) is removed we have the definition of unrestricted rectangular convergence. Henceforth, converge will mean converge unrestrictedly rectangularly. Explicitly, $T(\boldsymbol{x})$ converges to $s(\boldsymbol{x})$ if

(1.2) $s(x)=\lim _{\|M\| \rightarrow \infty} s_{M}(x) \quad$ where $s_{M}(x)=\sum_{m_{1}=-M_{1}}^{M_{1}} \sum_{m_{2}=-M_{2}}^{M_{2}} \ldots \sum_{m_{k}=-M_{k}}^{M_{k}} a_{m} e^{i m \cdot x}$

where $M=\left(M_{1}, \ldots, M_{k}\right)$. Note that convergence is not a regular method of convergence. The series $T(\boldsymbol{x})$ converges in the sense of Pringsheim to $s(\boldsymbol{x})$ if (1.2) 
holds and if there is a number $B=B(x)$ such that $\left|s_{M}(x)\right| \leqq B$ for all $\boldsymbol{M}$. For example, if $a_{0 n}=|n|, a_{1 n}=-|n|, a_{m n}=0$ otherwise, then $T(\mathbf{0})$ converges to 0 , but $s_{0, N}(0)$ $=N(N+1)$ so $T(\mathbf{0})$ does not converge in the sense of Pringsheim.

We will consider several summability methods. A series $T$ is spherically Abel summable to $s(\boldsymbol{x})$ if $T(\boldsymbol{x}, t)=\sum a_{\boldsymbol{m}} \exp (\mathrm{im} \cdot \boldsymbol{x}-|\boldsymbol{m}| t)$ converges absolutely for $t>0$ and if $\lim _{t \rightarrow 0} T(x, t)=s(x)$. We say $T$ is Riemann summable to $s(x)$ if

$$
T\left(\boldsymbol{x} ; h_{1}, \ldots, h_{k}\right)=\sum a_{m} e^{i m \cdot x}\left(\frac{\sin m_{1} h_{1}}{m_{1} h_{1}}\right)^{2}\left(\frac{\sin m_{2} h_{2}}{m_{2} h_{2}}\right)^{2} \ldots\left(\frac{\sin m_{k} h_{k}}{m_{k} h_{k}}\right)^{2}
$$

converges absolutely for $h_{1}^{2}+\cdots+h_{k}^{2}=|h|^{2} \neq 0$ (interpret ( $\left.\sin 0\right) / 0$ to be 1 ), and if $\lim _{|h| \rightarrow 0} T\left(x ; h_{1}, \ldots, h_{k}\right)=s(x)$.

We will consider only Lebesgue measurable subsets $A$ of $T^{k}$ and $|A|$ will denote the Lebesgue measure of $A$.

\section{Relationships between methods of convergence and the growth of coefficients.}

Lemma 2.1 (P. J. CoHen [2]). Let $S \subset T^{1}$ with $|S|>\delta$. Then for any integer $l>0$ there are l points of $S ; z_{1}, \ldots, z_{l}$, such that $\left|z_{i}-z_{j}\right|>(\delta / l)$ for $i \neq j$.

Proof. We choose $z_{p}, p=1,2, \ldots, l$, recursively as follows. Let $z_{1}$ be any point of $S$. For this proof only identify $T^{1}$ with $\left[z_{1}, z_{1}+2 \pi\right)$. Choose $z_{2}>z_{1}+\delta / l$ so that $\left|S \cap\left[z_{1}, z_{2}\right]\right|<|S| / l$. Having chosen $z_{2}, \ldots, z_{p-1}$ choose $z_{p}>z_{p-1}+\delta / l$ so that $\left|S \cap\left[z_{p-1}, z_{p}\right]\right|<|S| / l$. This can be done since

$$
\begin{aligned}
&\left|S \cap\left[z_{1}, z_{p-1}\right]\right|=\sum_{i=1}^{p-2}\left|S \cap\left[z_{i}, z_{i+1}\right]\right|<(p-2) \frac{|S|}{l} \\
& \Rightarrow\left|S \cap\left[z_{p-1}, 2 \pi\right)\right|>|S|-(p-2) \frac{|S|}{l}>2 \frac{\delta}{l} .
\end{aligned}
$$

Observe that

$$
\left|S \cap\left[z_{1}, z_{l}\right]\right|<\frac{l-1}{l}|S| \Rightarrow\left(z_{1}+2 \pi\right)-z_{l} \geqq\left|S \cap\left(z_{l}, z_{1}+2 \pi\right)\right| \geqq \frac{|S|}{l}>\frac{\delta}{l},
$$

so that $z_{1}$ and $z_{l}$ are sufficiently separated as points of $T^{1}$. It is clear from the construction that any other pair of points are also sufficiently separated.

LEMMA 2.2. Let $p(w)=a_{0}+a_{1} w+\cdots+a_{n} w^{n}$ be a trigonometric polynomial where $w=e^{i x}$ ranges over the unit circle.

(a) If $|p(w)| \leqq B$ for $w \in E \subset T^{1},|E|>0$, then there is a constant $c=c(|E|)$ such that $|p(w)| \leqq B c^{n}$ for all $w$ in $T^{1}$.

(b) If given any $\gamma>1$, there exists $\lambda=\lambda(\gamma)<1$ and $b=b(\gamma)$ such that $|p(w)| \leqq B$ on some set $E \subset T^{1},|E|>2 \pi \lambda$, then for all $w \in T^{1}$,

$$
|p(w)| \leqq B b \gamma^{n} .
$$

Proof. We will treat $\lambda \in(0,1)$ as a parameter. Its value will be determined later. In the proof of (a), $\lambda$ will be $|E| / 2 \pi$; in the proof of (b), $\lambda$ will depend on $\gamma$. Let 
$z_{0}, z_{1}, \ldots, z_{n}, n \geqq 1$, be points of $\{z \in C:|z|=1\}$ such that $\left|\arg z_{i}-\arg z_{j}\right|$ $\geqq 2 \pi \lambda /(n+1)$ if $i \neq j$. Since we will use the Lagrange interpolation formula $p(z)$ $=\sum_{i=0}^{n} p\left(z_{i}\right) \pi_{i}(z)$ where

$$
\pi_{i}(z)=\frac{\prod_{j \neq i}\left(z-z_{j}\right)}{\prod_{j \neq i}\left(z_{i}-z_{j}\right)},
$$

we need to estimate the $\pi_{i}(z)$. Because of symmetry, it suffices to estimate $\pi_{0}(z)$. Without loss of generality, we assume that

$$
0=\arg z_{0}<\arg z_{1}<\cdots<\arg z_{n}<2 \pi-2 \pi \lambda /(n+1)<2 \pi .
$$

Let $m$ be the smallest integer greater than $(n+1) / \lambda$ such that $m-n-1$ is an even integer. Note $m$ and $n$ have opposite parity. Let $\zeta_{1}=e^{2 \pi i / m}, \zeta_{-1}=e^{-2 \pi i / m}, \zeta_{2}=e^{2 \pi(2 / m) i}$, $\zeta_{-2}=e^{-2 \pi(2 / m) i}, \ldots, 1$ be the $m$ th roots of unity, indexed so that $\left|\zeta_{i}-1\right|<\left|\zeta_{j}-1\right|$ if $|i|<|j| \leqq m / 2$. Observe that

$$
\pi_{0}(z)=\frac{\prod_{j=1}^{n}\left(z-z_{j}\right)}{\prod_{j=1}^{n}\left(1-z_{j}\right)}
$$

Let $A=$ the set of the $n \zeta$ 's nearest to 1 (excluding 1 itself), $B=$ the set of the $n \zeta$ 's furthest from $1, B^{\prime}=$ the set of the $m-n-1 \zeta$ 's nearest to 1 (excluding 1 itself), $A^{\prime}=$ the set of the $m-n-1 \zeta$ 's furthest from 1 , and finally, $C=$ the set of all $\zeta$ 's except $\zeta=1$. Note $A \cup A^{\prime}=B \cup B^{\prime}=C, A \cap A^{\prime}=B \cap B^{\prime}=\varnothing$, the empty set. We have easily

$$
\left|\prod_{j=1}^{n}\left(1-z_{j}\right)\right| \geqq\left|\prod_{\zeta \in A}(1-\zeta)\right|
$$

for $\left|1-z_{1}\right| \geqq\left|1-\zeta_{1}\right|,\left|1-z_{n}\right| \geqq\left|1-\zeta_{-1}\right|,\left|1-z_{2}\right|>\left|1-\zeta_{-1}\right|,\left|1-z_{n-1}\right|>\left|1-\zeta_{-2}\right|$, etc.

We also have

$$
\left|\prod_{j=1}^{n}\left(z-z_{j}\right)\right| \leqq\left|\prod_{\zeta \in B}(1-\zeta)\right| .
$$

To give a formal argument for (2.5) is tedious while the essential idea is geometrically evident. We give a description of the argument. When $n$ is odd, one chooses $y$ to be a $z_{j}$ which is closest to $-z$.

By pushing the remaining $z_{j}$ 's closer to $y$ one obtains a new group of $z_{j}$ 's whose neighboring elements differ in argument by $2 \pi / m$ while the product corresponding to the left-hand side of (2.5) is increased.

Finally, a maximum is obtained when this new grouping is rigidly rotated so that $y$ moves to $-z$, giving a new product on the left-hand side of $(2.5)$ which is in modulus equal to the right-hand side. In the case that $n$ is even, one proceeds in a similar way so that the final rotation of the adjusted grouping of points has its two nearest points to $-z$ straddling $-z$ in the same way the two nearest $m$ th roots of unity nearest to -1 straddle -1 . 
From (2.3), (2.4), and (2.5) we have

$$
\left|\pi_{0}(z)\right| \leqq \frac{\prod_{\zeta \in B}|1-\zeta|}{\prod_{\zeta \in A}|1-\zeta|}=\frac{\prod_{\zeta \in C}|1-\zeta| / \prod_{\zeta \in B^{\prime}}|1-\zeta|}{\prod_{\zeta \in C}|1-\zeta| / \prod_{\zeta \in A^{\prime}}|1-\zeta|}=\frac{\prod_{\zeta \in A^{\prime}}|1-\zeta|}{\prod_{\zeta \in B^{\prime}}|1-\zeta|}
$$

Define $\delta$ by $\delta n=(m-n-1) / 2$. Then $\delta=\delta(\lambda, n)$ satisfies

$$
\frac{1}{2}\left(\frac{1}{\lambda}-1\right)<\frac{((n+1) / \lambda)-(n+1)}{2 n}<\delta<\frac{(n+1) / \lambda+2-(n+1)}{2 n} \leqq\left(\frac{1}{\lambda}-1\right)+\frac{1}{n}
$$

so that $\delta \rightarrow 0$ as $\lambda \rightarrow 1$ and $n \rightarrow \infty$.

Estimating each term in the numerator of (2.6) by 2, we have

$$
\prod_{\zeta \in A^{\prime}}|1-\zeta|<2^{2 \delta n}
$$

For the denominator we have

$$
\begin{aligned}
\prod_{\zeta \in B^{\prime}}|1-\zeta| & =\left|\prod_{k=1}^{\delta n}\left(1-e^{2 \pi i k / m}\right)\left(1-e^{-2 \pi i k / m}\right)\right|=\prod_{k=1}^{\delta n}\left(2 \sin \frac{k \pi}{m}\right)^{2}>\prod_{k=1}^{\delta n}\left(\frac{4 k}{m}\right)^{2} \\
& =(4 / m)^{2 \delta n}(\delta n) !^{2}>(4 / m)^{2 \delta n}\left((2 \pi)^{1 / 2}(\delta n)^{\delta n+1 / 2} e^{-\delta n}\right)^{2} .
\end{aligned}
$$

This last inequality follows from Stirling's inequality. Combining this with (2.6) and (2.8) we have

$$
\left|\pi_{0}(z)\right| \leqq(1 / 2 \pi \delta n)(e((2 \delta+1) n+1) / 2 \delta n)^{2 \delta n} .
$$

We now prove Lemma 2.2. Given $p(w)$ and $E \subset T^{1},|E|>2 \pi \lambda$, by Lemma 2.1 we may choose $z_{0}, z_{1}, \ldots, z_{n}$ from $E$ where $\left|\arg z_{i}-\arg z_{j}\right|>2 \pi \lambda /(n+1)$ if $i \neq j$. Assume $p$ is bounded by $B$ on $E$, and recall $p(z)=\sum_{i=0}^{n} p\left(z_{i}\right) \pi_{i}(z)$. By (2.9) and the comment following (2.2) we have, for all $z$,

$$
\begin{aligned}
|p(z)| & \leqq B \frac{n+1}{2 \pi}\left(\frac{e((2 \delta+1) n+1)}{2 \delta n}\right)^{2 \delta n} \frac{1}{\delta n} \\
& \leqq B\left\{\frac{1}{(\delta \pi)^{1 / n}}\left(\frac{e(2 \delta+1+1 / n)}{2 \delta}\right)^{2 \delta}\right\}^{n}=B\{\gamma(n, \delta)\}^{n} .
\end{aligned}
$$

In order to prove (a), we choose $\lambda=|E| / 2 \pi>0$ so that, by (2.7), $\delta>0$ is bounded away from zero. Hence, $\sup _{n, \delta} \gamma(n, \delta)=c$. To prove (b), first choose $\lambda$ so close to 1 and then $n_{0}$ so large that $\gamma(\dot{n}, \delta)>\gamma$ if $n \geqq n_{0}$. Set $b=\max _{k=1, \ldots, n_{0}-1}\left\{\gamma(\delta, k)^{k}, 1\right\}$. From (2.10) we have $|p(z)| \leqq B b \gamma^{n}$. This completes the proof of Lemma 2.2.

LEMMA 2.3. If $T(\boldsymbol{x})$ converges at each $\boldsymbol{x}$ of a set $E$ of positive measure (more generally, if $\lim \sup _{\| \boldsymbol{n} \mid \rightarrow \infty}\left|T_{n}(\boldsymbol{x})\right|<\infty$ for $\boldsymbol{x}$ in $E$ ), then there is a set $F \subset E,|F|=|E|$ such that all rectangular partial sums are bounded on $F$. (The bound may vary from point to point.) In particular, if $E=T^{k}$ (or even if the complement of $E$ is countable), then the conclusion holds everywhere on $E$.

Remark. To appreciate that this lemma is trivial only if $k=1$, consider the simple numerical series $s=\sum_{m, n=0}^{\infty} a_{m n}$ where $a_{0 n}=2^{n}, a_{1 n}=-2^{n}, a_{m n}=0$ otherwise. 
Here $s_{m n}=0$ if $m$ and $n$ are both greater than two so $s$ converges to 0 , but $s_{0 n}=2^{n+1}$ -1 so the partial sums are unbounded.

Proof. First let $k=2$. Let $F=\left\{\left(x_{0}, y_{0}\right) \in E\right.$ : the one-dimensional measures of $E_{x_{0}}=E \cap\left\{x=x_{0}\right\}$ and $E_{y_{0}}=E \cap\left\{y=y_{0}\right\}$ are both positive $\}$. It will suffice to show the partial sums bounded at each $\left(x_{0}, y_{0}\right) \in F$; since whenever the complement of $E$ is countable, $F=E$, and since $F$ is in any case a subset of $E$ of full measure (apply Fubini's theorem to the characteristic function of $E-F$ to see this). Given $\left(x_{0}, y_{0}\right)$ find $B$ and $\mu$ so large that

$$
\begin{aligned}
& \left|T_{m n}\left(x_{0}, y\right)\right| \leqq B \quad \text { whenever } m, n \geqq \mu \text { and } y \in F_{y_{0}}, \\
& \left|T_{m n}\left(x, y_{0}\right)\right| \leqq B \quad \text { whenever } m, n \geqq \mu \text { and } x \in F_{x_{0}},
\end{aligned}
$$

where $F_{x_{0}} \subset E_{x_{0}}$ and $F_{y_{0}} \subset E_{y_{0}}$ have positive measure. Further, choose $B$ so large that

$$
\left|T_{m n}\left(x_{0}, y_{0}\right)\right| \leqq B
$$

if $m$ and $n$ are both less than $\mu$. This can be done since (2.12) simply demands that $B$ be bigger than $(\mu+1)^{2}$ numbers. We still have to study $T_{m n}\left(x_{0}, y_{0}\right)$ when $m \geqq \mu>n$ or $n \geqq \mu>m$. The two cases are symmetrical; so henceforth, assume $m \geqq \mu>n$. From (2.11), $\left|T_{m \mu}\left(x_{0}, y\right)\right| \leqq B$ for all $y \in F_{y_{0}}$. Thinking of $T_{m \mu}$ as a polynomial in $e^{i y}$ of degree $2 \mu$ since

$$
\left|T_{m \mu}(x, y)\right|=\left|\sum_{\beta=-\mu}^{\mu} \sum_{\alpha=-m}^{m} t_{\alpha \beta} e^{i \alpha x} e^{i(\mu+\beta) y}\right|
$$

and applying Lemma 2.2 we obtain for all $y$

$$
\left|T_{m \mu}\left(x_{0}, y\right)\right| \leqq B c^{2 \mu}, \quad c=c\left(\left|E_{y_{0}}\right|\right) .
$$

Since a polynomial is its own Fourier series and a function's supremum dominates its Fourier coefficients, we obtain the same inequality for the coefficients

$$
\left|\sum_{\alpha=-m}^{m} t_{\alpha \beta} e^{i \alpha x_{0}}\right| \leqq B c^{2 \mu}, \quad \beta=-\mu,-\mu+1, \ldots, \mu .
$$

Use this inequality $2 n+1$ times to obtain

$$
\left|T_{m n}\left(x_{0}, y\right)\right| \leqq \sum_{\beta=-n}^{n} B c^{2 \mu}=(2 n+1) B c^{2 \mu}<(2 \mu+1) B c^{2 \mu} \text { for all } y .
$$

In particular, this last inequality holds for $y=y_{0}$, which was to be shown.

For the case of $k>2$, the theorem can be proved by an induction which requires no new ideas not already present in the $k=2$ case. One simply replaces $m$ by $\left(n_{1}, \ldots, n_{k-1}\right), n$ by $n_{k}$ and makes a similar decomposition of $F$.

For completeness we include a proof of the Cantor-Lebesgue Theorem. One could also see Reves and Szász [14, pp. 693-695] or Geiringer [8, p. 69]. For $\boldsymbol{n} \geqq 0, A_{n}(\boldsymbol{x})$ is the sum of all the elements of $\left\{a_{l} \exp [i \boldsymbol{i l} \cdot \boldsymbol{x}]:\left|l_{i}\right|=n_{i}, i=1, \ldots, k\right\}$. 
Cantor-Lebesgue Theorem. If $\lim _{\min n_{i} \rightarrow \infty} A_{n}(x)=0$ for all $x \in E,|E|>0$, then $\lim _{\|n\| \rightarrow \infty} a_{n}=0$.

Proof. We proceed by induction. For the theorem in one dimension see Zygmund [23, Vol. I, Chapter IX]. Assume the theorem holds for $k-1$ and that

$$
\text { Real } \begin{aligned}
\left(A_{\boldsymbol{n}}(\boldsymbol{x})\right) & =a_{\boldsymbol{n}}\left(\boldsymbol{x}^{\prime}\right) \cos n_{k} \boldsymbol{x}_{k}+b_{\boldsymbol{n}}\left(\boldsymbol{x}^{\prime}\right) \sin n_{k} \boldsymbol{x}_{k} \\
& =\rho\left(\boldsymbol{n} ; \boldsymbol{x}^{\prime}\right) \cos \left(n_{k} \boldsymbol{x}_{k}+\alpha\left(\boldsymbol{n} ; \boldsymbol{x}^{\prime}\right)\right)=o(1) \quad \text { as }\|\boldsymbol{n}\| \rightarrow \infty
\end{aligned}
$$

for every $\boldsymbol{x}=\left(\boldsymbol{x}^{\prime}, \boldsymbol{x}_{k}\right) \in E$. It suffices to show that $\rho\left(\boldsymbol{n} ; \boldsymbol{x}^{\prime}\right) \rightarrow 0$ for every $\boldsymbol{x}^{\prime}$ in some set $F$ of positive measure. Let $F=\left\{\boldsymbol{x}^{\prime}: \int_{-\pi}^{\pi} \chi_{E}\left(\boldsymbol{x}^{\prime}, x_{k}\right) d x_{k}>0\right\}$, where $\chi_{E}$ is the characteristic function of $E$. By Fubini's theorem, $|F|>0$. For any fixed $x^{\prime} \in F$,

$$
\rho^{2} \cos ^{2}\left(n_{k} x_{k}+\alpha\right) \rightarrow 0 \text { for all } x_{k} \in\left\{x_{k}:\left(x^{\prime}, x_{k}\right) \in E\right\}=F\left(x^{\prime}\right) \text {. }
$$

But by definition of $F,\left|F\left(x^{\prime}\right)\right|>0$, so integrating (2.16) over $F\left(x^{\prime}\right)$ shows that $\frac{1}{2} \rho^{2}\left|F\left(x^{\prime}\right)\right|$, and hence also $\rho$, tends to 0 as $\|\boldsymbol{n}\| \rightarrow \infty$.

THEOREM 2.1. If $T(\boldsymbol{x})$ is convergent almost everywhere by a regular method of convergence of eccentricity $k$, and if $\gamma>1$ is given, then there exists $b=b(T, \gamma, k)>0$ such that

$$
\left|a_{m}\right| \leqq b \gamma\|m\| .
$$

If $T(x)$ is convergent on a set $E$ of positive Lebesgue measure $|E|$, then there is $c=c(T,|E|, k)>1$ such that

$$
\left|a_{m}\right| \leqq c\|m\|
$$

These results are in a sense best possible.

Proof. Let $\gamma>1$ be given. Let $s_{n}(x)=\sum_{j \in E_{n}} c_{j} e^{i j \cdot x}$ be the $n$th partial sum of $T(x)$ with respect to the given regular method of summation. We are given that $\left\{s_{n}(x)\right\}$ converges for almost every $\boldsymbol{x} \in T^{k}$; hence, that the $s_{n}(\boldsymbol{x})$ are uniformly bounded on arbitrary large subsets of $T^{k}$. Consider $s_{n}(x)$ as a polynomial in one complex variable $\exp \left(i x_{1}\right)=z_{1}$ with coefficients depending on $\boldsymbol{x}^{\prime}=\left(x_{2}, \ldots, x_{k}\right)$,

$$
\left|s_{n}\left(x_{1}, \boldsymbol{x}^{\prime}\right)\right|=\left|\sum_{j=-J_{1}}^{J_{1}} d_{j}\left(\boldsymbol{x}^{\prime}\right) \exp \left(i j x_{1}\right)\right|=\left|\sum_{m=0}^{2 J_{1}} d_{m-J_{1}}\left(\boldsymbol{x}^{\prime}\right) \exp \left(i m x_{1}\right)\right| .
$$

We may assume that $s_{n}$ is bounded by a constant $B_{\lambda}$ on a set of the form $\bigcup_{x^{\prime} \in H}\left(G\left(x^{\prime}\right) \times\left\{x^{\prime}\right\}\right)$ where $|H|>(2 \pi \lambda)^{k-1}$; for every $\boldsymbol{x}^{\prime} \in H,|G(\boldsymbol{x})|>(2 \pi \lambda)$ and $\lambda<1$ may be chosen arbitrarily close to 1 . Fix any $\boldsymbol{x}^{\prime} \in H$; by Lemma 2.2, part (b), $\left|s_{n}\left(x_{1}, \boldsymbol{x}^{\prime}\right)\right| \leqq B_{\lambda} b\left(\gamma_{1}\right) \gamma_{1}^{2 s_{1}}$ for every $x_{1}$ so that by using Fourier's integral representation for the coefficients of the polynomial as in the proof of Lemma 2.3, we obtain

$$
\left|d_{j}\left(\boldsymbol{x}^{\prime}\right)\right|=\left|\frac{1}{2 \pi} \int_{0}^{2 \pi} s_{n}(\boldsymbol{x}) \exp \left(-i j x_{1}\right) d x_{1}\right| \leqq B_{\lambda} b\left(\gamma_{1}\right) \gamma_{1}^{2 J_{1}}, \quad \boldsymbol{x}^{\prime} \in H .
$$


If we apply the same argument to the $d_{j}$, if the original $\lambda$ was sufficiently close to 1 , a $k$-fold iteration yields

$$
\left|c_{j}\right| \leqq B_{\lambda}\left(b\left(\gamma_{1}\right)\right)^{k} \gamma_{1}^{2\left(J_{1}+\cdots+J_{k}\right)} \quad \text { for each } \boldsymbol{j} \in E_{n} .
$$

But by the definition of regularity, given any $\boldsymbol{j}$, there is an

$$
E_{n} \subset\left\{-J_{1},-J_{1}+1, \ldots, J_{1}\right\} \times \cdots \times\left\{-J_{k}, \ldots, J_{k}-1, J_{k}\right\}
$$

such that $\|\boldsymbol{j}\| K \geqq \sup _{i=1, \ldots, k} J_{i}, j \in E_{n}$. Hence,

$$
\left|c_{j}\right| \leqq B_{\lambda}\left(b\left(\gamma_{1}\right)\right)^{k} \gamma_{1}^{2 k K \|}\|j\| .
$$

If we set $\gamma_{1}=\gamma^{1 / 2 k K}$ and $b=B_{\lambda} b\left(\gamma_{1}\right)^{k}$, this proves the first part of Theorem 2.1.

The second part of Theorem 2.1 follows exactly the same line of proof except that part (a) of Lemma 2.2 is used in place of part (b).

P. J. Cohen [2, p. 44] gives examples of trigonometric series whose coefficients are "almost" $O(\|\boldsymbol{m}\|)$. To show that Theorem 2.1 is best possible we give new examples. Consider a two dimensional series of the form

$$
t(x, y)=\sum_{n=1}^{\infty} \phi(n)(1-\cos x)^{n} e^{i n y}
$$

where the choice of $\phi$ will determine the properties of $t$. Since

$$
(1-\cos x)^{n}=\left(2 \sin \frac{x}{2}\right)^{2 n}=2^{-n} \sum_{j=0}^{2 n}\left(\begin{array}{c}
2 n \\
j
\end{array}\right) e^{i(n-j) x}(-1)^{n-j},
$$

the $N$ th partial sum of (2.19) may be written

$$
t_{N}(x, y)=\sum_{n=1}^{N} \sum_{m=-n}^{n}(-1)^{m} \phi(n) 2^{-n}\left(\begin{array}{c}
2 n \\
n+m
\end{array}\right) e^{i m x} \cdot e^{i n y}=\sum_{n=-N}^{N} \sum_{m=-N}^{N} t_{m n} e^{i(m x+n y)}
$$

From (2.21) it is clear that the ordinary $N$ th partial sum of $t$ corresponds to the $(N, N)$ square partial sum of $t$ thought of as a double series. We note that

$$
\begin{aligned}
\left|t_{0, n}\right| & =\phi(n) 2^{-n}\left(\begin{array}{c}
2 n \\
n
\end{array}\right)=\phi(n) 2^{-n}\left(\frac{(2 n) !}{n !^{2}}\right) \\
& >\phi(n) 2^{-n}\left(\frac{(2 \pi(2 n))^{1 / 2}(2 n)^{2 n} e^{-2 n}}{\left((5 / 4)(2 \pi n)^{1 / 2} n^{n} e^{-n}\right)^{2}}\right)>\phi(n) 2^{-n}\left(\frac{2^{2 n}}{3 n^{1 / 2}}\right)=\frac{\phi(n)}{3 n^{1 / 2}} 2^{n} .
\end{aligned}
$$

Now set $\phi(n)=3 n^{1 / 2}$. For each $x$ satisfying $|1-\cos x|<1$ and for all $y$, that is for $(x, y) \in(-\pi / 2, \pi / 2) \times[-\pi, \pi]$, we have that $t_{N, N}$ converges absolutely. However, $\left|t_{0, n}\right|>2^{n}$ which shows that (2.18) cannot be improved. To see that the "almost everywhere" hypothesis in the first part of Theorem 2.1 cannot be weakened, set $\phi(n)=3 n^{1 / 2} r^{-n}$. Given any $\varepsilon>0, r<2$ may be chosen so close to 2 as to make the set of convergence of $t$ have measure $>(2 \pi)^{2}-\varepsilon$. Nevertheless, $\left|t_{0, n}\right|>(2 / r)^{n}$, so that the coefficients still grow exponentially.

Finally, given that a series does converge almost everywhere with respect to some regular method, the somewhat awkward condition (2.17) is the most that one 
can deduce concerning the growth of its coefficients. Let $N=\|(m, n)\|$ $=\max \{|m|,|n|\}$. Assume that $t_{m, n}$ is square convergent almost everywhere. Then (2.17) asserts that

$$
\text { for all } \gamma>1, \quad\left|t_{m, n}\right| / \gamma^{N}<b_{\gamma}
$$

where $b_{\gamma}$ is independent of $m$ and $n$. This is equivalent to

$$
\text { for all } \gamma>1, \lim _{N \rightarrow \infty} \frac{\left|t_{m, n}\right|}{\gamma^{N}}=0 \text {. }
$$

For (2.24) obviously implies (2.23) and conversely assuming (2.23) and given $\gamma>1$, we have, picking $\gamma^{\prime}$ between $\gamma$ and 1 ,

$$
\frac{\left|t_{m, n}\right|}{\gamma^{N}}=\frac{\left|t_{m, n}\right|}{\gamma^{\prime N}}\left(\frac{\gamma^{\prime}}{\gamma}\right)^{N} \leqq b_{r} \cdot o(1)
$$

Now given any function $\psi(N)$ such that for all $\gamma>1, \psi(N) / \gamma^{N} \rightarrow 0$, choose $\phi(n)=3 n^{1 / 2} \psi(n) 2^{-n}$. Then almost everywhere (in fact except for the two lines $x=\pi$ and $x=-\pi), t(x, y)$ converges as can be seen by setting $\gamma^{2}=2 /(1-\cos x)$ if $x \not \equiv \pi$ (if $x \equiv 0, t(0, y)=0$ ). But $\left|t_{0, n}\right|>\psi(n)$ so the coefficients grow faster than $\psi$. For concrete examples the reader might consider $\psi(n)=n^{10^{6}}$ or $\psi(n)=2^{n /(\log n)}$. In other words, almost everywhere square convergence permits the coefficients any rate of growth which is less than exponential.

REMARK. Lemma 2.2 is also fairly sharp. Let $P(z)=e^{i n x}((1-\cos x) / 2)^{n} . P$ is a polynomial of degree $2 n$. If $|P(z)| \leqq B$ on $[-\delta, \delta], B \simeq(\delta / 2)^{2 n}$ and $\sup |P(z)|=1$ so that $\sup |P(z)| \simeq B(4 /|[-\delta, \delta]|)^{2 n}$. This shows that the $c=c(|S|)$ of Lemma 2.2 cannot be chosen smaller than $4 /|S|$. A close reading of the proof of Lemma 2.2 shows that $c$ may be chosen of the form $d /|S|$ but we do not know if $d$ may be as small as 4 .

THEOREM 2.2. If $T(x)$ is convergent on a set $E,|E|>0$, then

$$
\begin{gathered}
a_{m}=o(1) \text { as }\|m\| \rightarrow \infty \text { and } \\
a_{m}=O(1) \text { for all } m .
\end{gathered}
$$

This is also a best possible result.

Proof. Given a numerical series $\sum_{i \geqq 0} a_{i}$, with partial sums $s_{n}=\sum_{n \geqq i \geqq 0} a_{i}$, it can be seen that

$$
a_{n}=\sum_{\delta \in \Omega^{k}}(-1)^{\delta} S_{n-\delta}
$$

In this formula $(-1)^{\delta}=(-1)^{\delta_{1}+\delta_{2}+\cdots+\delta_{k}}$ and we understand $s_{n-\delta}$ to be equal to 0 whenever any $n_{i}-\delta_{i}=-1$. In one and two dimensions this reduces to the elementary facts that $a_{n}=s_{n}-s_{n-1}$ and $a_{m, n}=s_{m, n}-s_{m-1, n}-s_{m, n-1}+s_{m-1, n-1}$.

The last equation may be visualized as $A=(A+B+C+D)-(B+D)-(C+D)$ $+D$ where $A=a_{m n}, B=\sum_{k=0}^{m-1} a_{k n}, C=\sum_{l=0}^{n-1} a_{m l}$ and $D=\sum_{k=0}^{m-1} \sum_{l=0}^{n-1} a_{k l}$. 


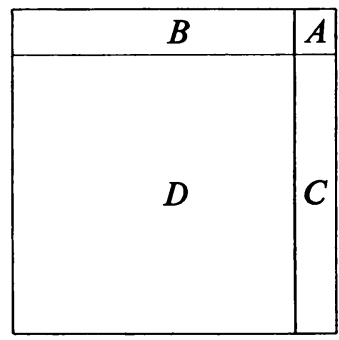

FIGURE 1

Since this figure somewhat resembles a Mondrian painting [5, p. 169], we will refer to an application of (2.27) as Mondrianing.

Let $A_{j}(\boldsymbol{x})=\sum_{\left|l_{i}\right|=j_{i}} c_{l} e^{i l \cdot x}$ where $\boldsymbol{j} \geqq 0$. If $T$ converges at $\boldsymbol{x}$ to $s$, writing

$$
T_{n}(x)=\sum_{n \geqq j \geqq 0} A_{j}(x),
$$

expressing $A_{n}(\boldsymbol{x})$ in terms of partial sums as in (2.27), observing that if all indices are large every partial sum in (2.27) is close to $s$ and that (2.27) has an equal number of positively and negatively signed terms, we find that

$$
\lim _{\min n_{i} \rightarrow \infty} A_{n}(x)=0 .
$$

From the hypothesis of Theorem 2.2, it follows that (2.28) holds for every $\boldsymbol{x}$ in a set of positive measure. From (2.28) and the Cantor-Lebesgue Theorem it follows that $\lim _{\|n\| \rightarrow \infty} a_{n}=0$, which is (2.25).

We now prove (2.26). From Lemma 2.3 and (2.27) we have that for each $x$ of $F$, there is a constant $C(x)$ such that

$$
\left|A_{j}(\boldsymbol{x})\right| \leqq C(x) \text { for all } \boldsymbol{j} \geqq 0 .
$$

Since $F$ has positive measure we may find a subset $F^{\prime} \subset F$ of positive measure on which (2.29) holds uniformly, that is,

$$
\left|A_{j}(\boldsymbol{x})\right| \leqq C \quad \forall \boldsymbol{x} \in F^{\prime},\left|F^{\prime}\right|>0 .
$$

From this it follows by the same argument used in the proof of the CantorLebesgue Theorem that we must also have

$$
\left|C_{j}\right| \leqq D \text { for all } j
$$

which is statement (2.26).

It remains to discuss the "best possible" aspects of Theorem 2.2. It is well known that (2.25) is best possible even in one dimension. In fact, a one-dimensional trigonometric series with coefficients going to zero arbitrarily slowly but which is nonetheless convergent almost everywhere may easily be found. For example,

$$
\sum_{k=1}^{\infty} \frac{\exp \left(i_{k} x\right)}{k}
$$


is the Fourier series of an $L^{2}$ function (since $\sum_{k=1}^{\infty} k^{-2}$ converges) and so convergent almost everywhere (Carleson [1]), but the $n_{k}$ may be chosen as rapidly increasing as desired, thus allowing (2.32) to have arbitrarily slowly decreasing coefficients.

EXAMPLE 2.1. Let $M(x)=\sum_{-\infty}^{\infty} d_{m} e^{i m x} \not \equiv 0$ be a one-dimensional trigonometric series which converges to zero outside of a set $M$, where $M$ is a set of multiplicity with $|M|=0$. For the construction of such an $M$ see [23, Vol. I, Chapter IX, §6]. Let $\delta(y)=\sum_{-\infty}^{\infty} e^{i n y}$ and let

$$
L(x, y)=M(x) \delta(y)
$$

Then

$$
L_{M, N}(x, y)=\sum_{m, n=-M,-N}^{M, N} l_{m n} e^{i(m x+n y)}=\sum_{m=-M}^{M} d_{m} e^{i m x} \frac{\sin \left(N+\frac{1}{2}\right) y}{\sin (y / 2)} .
$$

Since whenever $x \notin M$ and $y \not \equiv$ the first factor tends to 0 , and the second factor is bounded by $\csc (y / 2), L(x, y)$ converges to zero for almost every $(x, y)$. However, if $d_{k}$ is any nonzero coefficient of $M(x)$, then $l_{k n}=d_{k}$ for every $n$ so that although the coefficients of $L$ are bounded, they do not tend to 0 as the second index increases. This shows that (2.26) cannot be improved.

REMARK. We can deduce that $c_{n} \rightarrow 0$ as $\|\boldsymbol{n}\| \rightarrow \infty$ if we start with a different mode of convergence. If $T(x)=\sum c_{n} e^{i n \cdot x}$ converges on a set $E$ of positive measure with respect to every regular method of convergence, then $\lim _{\|n\| \rightarrow \infty} c_{n}=0$. Let $k=2$ for simplicity. In particular, $T$ converges with respect to rectangles with sides parallel to the $m$ and $n$ axes and of eccentricity $\leqq 3$ and also with respect to rectangles with sides parallel to the lines $m=n$ and $m=-n$ and of eccentricity $\leqq 3$. By Mondrianing the former we get

$$
A_{m, n}(x)=a_{m, n} e^{i(m x+n y)}+a_{-m, n} e^{i(-m x+n y)}+a_{m,-n} e^{i(m x-n y)}+a_{-m,-n} e^{i(-m x-n y)} \rightarrow 0
$$

if $\frac{1}{3}<|m| n \mid<3$. Mondrianing the latter yields $A_{m, n}(\boldsymbol{x}) \rightarrow 0$ whenever $(m, n)$ is near an axis and, in particular, whenever $|m / n| \geqq 3$ or $|m / n| \leqq \frac{1}{3}$. Hence, $\lim _{\max \{m, n\} \rightarrow \infty} A_{m, n}(\boldsymbol{x})=0$ for all $\boldsymbol{x}$ in $E$. By the Cantor-Lebesgue Theorem, $\lim _{\|(m, n)\| \rightarrow \infty} a_{m, n}=0$.

It has recently been shown by $R$. Cooke [3] that the above result holds for series in two variables and circular convergence on a set of full measure. Subsequently, A. Zygmund [25] has reduced his hypothesis to circular convergence on a set of positive measure. Neither result has been extended to $k>2$, at this writing.

\section{Relationships between modes of convergence and summability.}

THEOREM 3.1. If

$$
T(x)=\sum c_{m} e^{i m \cdot x}
$$

converges at each $\boldsymbol{x}$ of a set $E$, then $T(\boldsymbol{x})$ is spherically Abel summable at almost every $\boldsymbol{x}$ in $E$. In particular, if the complement of $E$ is empty (or even countable), then the conclusion holds everywhere on $E$. 
Proof. An application of Lemma 2.3 shows that it is sufficient to prove the following lemma concerning numerical series.

LEMMA 3.1. If $\sum_{m \geqq 0} c_{m}$ converges to $s$ and has all partial sums bounded, then it is spherically Abel summable to $s$; that is, $\sum_{m \geqq 0} c_{m} e^{-|m| h}=s(h)$ exists for every $h>0$ and $\lim _{h \rightarrow 0} s(h)=s$.

This lemma is, in turn, a consequence of the following lemma of standard type.

Before we state the lemma we must develop some additional notation. Let $p \in \Omega^{k}$ with 1's in the $i_{1}, i_{2}, \ldots, i_{r}$ places and 0 's in the remaining $k-r$ places. Then

$$
\sum_{(p)} a_{m}=\sum_{m_{1}=0}^{\infty} \sum_{m_{t_{2}}=0}^{\infty} \cdots \sum_{m_{i_{r}}=0}^{\infty} a_{m}
$$

where $m_{j}$ is fixed if $j \notin\left\{i_{1}, i_{2}, \ldots, i_{r}\right\}$. In particular, if $p=\mathbf{0}, \sum_{(x)} a_{m}=a_{m}$. Further,

$$
\sum_{(p)}^{\prime} a_{m}=\sum_{i_{1}=0}^{\infty} \cdots \sum_{m_{i_{s}-1}=0}^{\infty} \sum_{m_{i_{s}+1}=0}^{\infty} \cdots \sum_{m_{i_{r}}=0}^{\infty} a_{m}
$$

where the prime indicates that one of the summations has been omitted. If $p=\mathbf{0}$, set $\sum_{(p)}^{\prime} a_{m}=0$. Note that if $p \neq 0$ and if all the $m_{j}$ with $j \notin\left\{i_{1}, \ldots, i_{r}\right\}$ are fixed, $\sum_{(p)} a_{m}$ denotes a single sum while $\sum_{(p)}^{\prime} a_{m}$ denotes an infinite family of sums, one for each choice of $s$ and $m_{i_{s}}, s=1,2, \ldots, r, m_{l_{s}}=0,1,2, \ldots$ If $p \neq 0$, we define $\Delta^{p} \lambda_{m}$ to be $\Delta_{i_{1}} \Delta_{i_{2}} \cdots \Delta_{i_{r}} \lambda_{m}$ where $\Delta_{i} \lambda_{m}=\lambda_{m_{1} \cdots m_{i-1} m_{i} m_{i+1} \cdots m_{k}}-\lambda_{m_{1} \cdots m_{i-1}, m_{i}+1, m_{i+1} \cdots m_{k}}$ and $\Delta^{0} \lambda_{m}=\lambda_{m}$.

LeMma 3.2. Let $\sum_{m \geqq 0} c_{m}$ converge to $s$ and have all partial sums bounded. Then $\sum c_{m} \lambda_{m}(h)$ exists for all $h>0$ and $\lim _{h \rightarrow 0} \sum c_{m} \lambda_{m}(h)=s$ provided the functions $\lambda_{m}(h)$ satisfy

$$
\sum_{(p)}\left|\Delta^{p} \lambda_{m}(h)\right| \leqq c
$$

for each $p \in \Omega^{k}$, where $c$ is independent of both $h(h>0)$ and of the values of the $m_{j}$ 's corresponding to the indices not summed;

$$
\lim _{h \rightarrow 0} \sum_{(p)}^{\prime}\left|\Delta^{p} \lambda_{m}(h)\right|=0
$$

for each fixed $p \in \Omega^{k}$, fixed choice of omitted summation, and fixed set of $m_{j}$ 's corresponding to the indices not summed; and

$$
\lambda_{0}(h)=1 \text {. }
$$

Proof. The proof is a direct application of Abel's partial summation formula:

$$
\sum_{i=1}^{k} \sum_{n_{i}=0}^{N_{i}+1} a_{n} \lambda_{n}=\sum_{p \in \Omega^{k}} \sum_{n_{i_{1}}=0}^{N_{i_{1}}} \sum_{n_{i_{2}}=0}^{N_{i_{2}}} \cdots \sum_{n_{i_{r}}=0}^{N_{i_{r}}} s_{m} \Delta^{p} \lambda_{m} .
$$

Here $s_{m}=\sum_{m \geqq u \geqq 0} a_{u}$ where $m_{i_{1}}=n_{i_{1}}, \ldots, m_{i_{r}}=n_{i_{r}}, m_{j}=N_{j}+1$ if $j \notin\left\{i_{1}, \ldots, i_{r}\right\}$.

We apply (3.3) with $a_{m}=c_{m}, \lambda_{m}=\lambda_{m}(h)$. We are given

$$
\left|s_{m}\right| \leqq B .
$$


Given any $\varepsilon>0$ there is a number $\mu$ such that

$$
\left|s_{m}-s\right|<\varepsilon /\left(2 \cdot 2^{k} c\right) \text { if }\|m\| \geqq \mu
$$

where $c$ is the constant of (3.2a).

In (3.3) if we put $a_{0}=1, a_{m}=0$ for $\boldsymbol{m} \neq \mathbf{0}$, we obtain

$$
1=\lambda_{0}(h)=\sum_{(p)} \sum_{n_{i_{1}}=0}^{N_{1}} \cdots \sum_{n_{i_{r}}=0}^{N_{r}} \Delta^{p} \lambda_{m}(h) .
$$

This follows since (3.2c) holds and $s_{m}=1$ for every $m$. By virtue of (3.6) we may assume $s=0$ and replace (3.5) by

$$
\left|s_{m}\right|<\varepsilon /\left(2 \cdot 2^{k} c\right) \text { if }\|\boldsymbol{m}\| \geqq \mu .
$$

Now

$$
\begin{aligned}
\mid \sum_{n_{1}=0}^{N_{1}} \ldots & \sum_{n_{i_{r}}=0}^{N_{r}} s_{m} \Delta^{p} \lambda_{m} \mid \\
\leqq & B \sum_{n_{i_{1}}=0}^{\mu-1}\left[\sum_{n_{i_{2}}=0}^{\infty} \cdots \sum_{n_{i_{r}}=0}^{\infty}\left|\Delta^{p} \lambda_{m}(h)\right|\right]+B \sum_{n_{i_{2}}=0}^{\mu-1}\left[\sum_{n_{i_{1}}=0}^{\infty} \cdots \sum_{n_{i_{r}}=0}^{\infty}\left|\Delta^{p} \lambda_{m}(h)\right|\right] \\
& +\cdots+B \sum_{n_{i_{r}=1}}^{\mu-1}\left[\sum_{n_{i_{1}}=0}^{\infty} \ldots \sum_{n_{i_{r}-1}=0}^{\infty}\left|\Delta^{p} \lambda_{m}(h)\right|\right] \\
& +\frac{\varepsilon}{2} \frac{1}{2^{k} c} \sum_{n_{i_{1}}=\mu}^{\infty} \sum_{n_{i_{2}}=\mu}^{\infty} \ldots \sum_{n_{i_{r}}=\mu}^{\infty}\left|\Delta^{p} \lambda_{m}(h)\right| .
\end{aligned}
$$

Each term in brackets may be chosen $<\varepsilon /\left(2 \cdot 2^{k} \cdot \mu r B\right)$ if $h$ is chosen small enough (say $h<h_{0}$ ) by (3.2b). Applying (3.2a) to the last term in (3.8) yields

$$
\left|\sum_{n_{i 1}=0}^{N_{1}} \ldots \sum_{n_{i_{r}}=0}^{N_{r}} s_{m} \Delta^{p} \lambda_{m}\right|<\frac{\varepsilon}{2} \frac{1}{2^{k}} \frac{1}{r}+\frac{\varepsilon}{2} \frac{1}{2^{k}} \frac{1}{r}+\cdots+\frac{\varepsilon}{2} \frac{1}{2^{k}} \frac{1}{r}+\frac{\varepsilon}{2} \frac{1}{2^{k}}=\varepsilon \cdot \frac{1}{2^{k}} .
$$

We also observe that

$$
\left|s_{N_{1}+1 \cdots N_{k}+1} \lambda_{N_{1}+1 \cdots N_{k}+1}(h)\right|<\frac{\varepsilon}{2} \cdot \frac{1}{2^{k} c} \cdot c<\varepsilon \cdot \frac{1}{2^{k}} \quad \text { if all } N_{i} \geqq \mu .
$$

Combining (3.9) if $p \neq \mathbf{0}$ and (3.10) if $p=\mathbf{0}$ with Abel's partial summation formula, we see that if $\varepsilon>0$ is given, one may choose first $\mu=\mu(\varepsilon)$ sufficiently large and then $h_{0}=h_{0}(\mu, \varepsilon)=h_{0}(\varepsilon)$ sufficiently small so that, whenever $N_{1}, \ldots, N_{k} \geqq \mu$ and $0<h<h_{0}(\varepsilon)$,

$$
\left|\sum_{i=1}^{k} \sum_{n_{i}=0}^{N_{i}+1} c_{m} \lambda_{m}(h)\right|<\varepsilon .
$$

This completes the proof of Lemma 3.2. Note that the proof shows that (3.2c) could have been replaced by the slightly more general

$$
\lim _{h \rightarrow 0} \lambda_{0}(h)=1 \text {. }
$$


To prove Lemma 3.1 it remains only to show that $\lambda_{m}(h)=e^{-|m| h}$ satisfies (3.2). It is immediate that $\lambda_{0}(h)=1$ so $(3.2 \mathrm{c})$ holds. To ease notation let

$$
Q=(1,1, \ldots, 1,0,0, \ldots, 0)
$$

have its first $r$ entries equal to 1 and its remaining $k-r$ entries equal to 0 . We must show

$$
\sum_{n_{1}=0}^{N_{1}} \cdots \sum_{n_{r}=0}^{N_{r}}\left|\Delta^{Q} \lambda_{n_{1} \cdots n_{r} N_{r}+1}+1 \cdots N_{k}+1\right| \leqq C
$$

and, say, that

$$
\sum_{n_{1}=0}^{N_{1}} \cdots \sum_{n_{r}=0}^{N_{r}-1}\left|\Delta^{Q} \lambda_{n_{1} \cdots n_{r-1} n_{r} N_{r+1}+1 \cdots N_{k}+1}(h)\right| \rightarrow 0 \quad \text { as } h \rightarrow 0
$$

for each fixed choice of $n_{r}, N_{r+1}, \ldots$, and $N_{k}$.

Let $f(x)=e^{-|x|}$ so that $f(h m)=\lambda_{m}(h)$. Then

$$
\Delta_{1} \lambda_{m}(h)=\int_{m_{1} h}^{\left(m_{1}+1\right) h} \frac{\partial f}{\partial x_{1}}\left(x_{1}, m_{2} h, \ldots, m_{k} h\right) d x_{1}, \ldots
$$

and

$$
\Delta^{Q} \lambda_{m}(h)=\int_{m_{1} h}^{\left(m_{1}+1\right) h} \cdots \int_{m_{r} h}^{\left(m_{r}+1\right) h}\left(\frac{\partial}{\partial x}\right)^{Q} f\left(x_{1}, \ldots, x_{r}, m_{r+1} h, \ldots, m_{k} h\right)(d x)^{Q}
$$

An easy induction shows that

$$
(\partial / \partial x)^{Q} f(x) \leqq C\left(1+|x|^{-(r-1)}\right) e^{-|x|}
$$

Hence, the left-hand side of (3.11) may be majorized by

$$
C \int_{0}^{\infty} \cdots \int_{0}^{\infty}\left(1+|x|^{-(r-1)}\right) e^{-|x|}(d x)^{Q}
$$

where $\boldsymbol{x}=\left(x_{1}, \ldots, x_{r},\left(N_{r+1}+1\right) h, \ldots,\left(N_{k}+1\right) h\right)$.

Let $p^{2}=x_{1}^{2}+\cdots+x_{r}^{2}, \quad c^{2}=\left(\left(N_{r+1}+1\right) h\right)^{2}+\cdots+\left(\left(N_{k}+1\right) h\right)^{2}$ and transform (3.15) to polar coordinates. Since the integration depends only on $p$, the $r-1$ angular integrations may be carried out separately so that (3.15) becomes

$$
D \int_{0}^{\infty} \frac{1+\left(p^{2}+c^{2}\right)^{-(r-1) / 2}}{\exp \left(\left(p^{2}+c^{2}\right)^{1 / 2}\right)} p^{r-1} d p<D \int_{0}^{\infty}\left(p^{r-1}+1\right) e^{-p} d p<\infty
$$

The proof of (3.12) is similar for we must now show that

$$
\int_{0}^{\infty} \cdots \int_{0}^{\infty} \int_{n_{r} h}^{\left(n_{r}+1\right) h}\left(\frac{\partial}{\partial x}\right)^{Q} f\left(x_{1}, \ldots, x_{r},\left(N_{r+1}+1\right) h, \ldots,\left(N_{k}+1\right) h\right)(d x)^{Q}
$$

which majorizes the left-hand side of (3.12) tends to 0 with $h$. 
Given $\varepsilon>0$, choose $h$ so small that $\left(n_{r+1}+1\right) h<\varepsilon$ and $c^{2}=\left(\left(N_{r+1}+1\right) h\right)^{2}+\cdots$ $+\left(\left(N_{k}+1\right) h\right)^{2}<1$. Then the integral corresponding to (3.16) is majorized by

$$
C \int_{0}^{\infty} \cdots \int_{0}^{\infty} \int_{0}^{\varepsilon} \frac{1+\left(p^{2}+1\right)^{-(r-1) / 2}}{e^{p}}(d x)^{Q},
$$

where $p^{2}=x_{1}^{2}+\cdots+x_{r}^{2}$. Transforming to polar coordinates shows the integrand to be integrable over $[0, \infty)^{r}$. A simple argument involving the definition of improper integrals then shows that (3.18) tends to 0 with $\varepsilon$ and hence $h$. This proves Theorem 3.1.

Remarks. Theorem 3.1 shows spherical Abel summability to be quite powerful. The theorem is somewhat surprising since different methods of convergence are quite incompatible. We consider now some examples which illustrate this incompatibility. All of our examples will be two dimensional.

EXAMPLE 3.1. There is a series which is square convergent at almost every point but which fails to converge restrictedly rectangularly at any point.

Consider the series

$$
a(x, y)=\sum_{l=2}^{\infty} 3 l \exp \left(i l^{2} x\right) \sin ^{2 l} y \exp \left(i\left(l^{2}-2 l\right) y\right)=\sum_{m, n=-\infty}^{\infty} a_{m, n} e^{i(m x+n y)} .
$$

Whenever $|\sin y|<1$, which is to say at almost every point $(x, y)$, the series converges absolutely. From the expansion

$$
\sin ^{2 l} y=(2 i)^{-2 l} \sum_{j=0}^{2 l}\left(\begin{array}{c}
2 l \\
j
\end{array}\right) e^{2 i y(l-j)}
$$

it follows that the partial sums of $a(x, y)$ coincide with the square partial sums of $a(x, y)$ thought of as a double series so that $a(x, y)$ is square convergent almost everywhere.

Let $e$ be any number greater than one. We will show that $a(x, y)$ is not restrictedly rectangularly convergent with eccentricity $e$. The ratios of the coordinates of $\left(l^{2}+\gamma, l^{2}-2 l+\delta\right)$ where $\gamma$ and $\delta$ may be 0 or -1 tend to one as $l$ tends to infinity. In particular, these ratios become less than $e$ if $l$ is sufficiently large. If $a(x, y)$ converges with eccentricity $e$ at some point $\left(x_{0}, y_{0}\right)$, Mondrianing (see (2.27)) shows that

$$
\left|A_{l^{2}, l^{2}-2 l}\left(x_{0}, y_{0}\right)\right|=\left|a_{l^{2}, l^{2}-2 l} \exp \left(i l^{2} x_{0}+\left(l^{2}-2 l\right) y_{0}\right)\right|=\left|a_{l^{2}, l^{2}-2 l}\right|
$$

tends to zero as $l$ tends to infinity. But from (3.19) and (3.20) (see also (2.22))

$$
\left|a_{l^{2}, l^{2}-2 l}\right|=\left|3 l(2 i)^{-2 l}\left(\begin{array}{c}
2 l \\
l
\end{array}\right)\right|>l^{1 / 2}
$$

which does not tend to zero.

Another interesting example due to C. Fefferman [6] asserts that there exists a continuous function of two variables which has a Fourier series which does not converge almost everywhere for restricted rectangular sums. This example also 
separates square and restricted rectangular convergence, since Fefferman [7], P. Sjolin [19], and N. Tevzadze [20] have shown that the Fourier series of a function in $L^{2}$ converges almost everywhere for square sums. One should note that $a(x, y)$ is not a Fourier series since by (3.22) the $a_{m, n}$ do not tend to zero.

EXAMPLE 3.2. There is a series which is restrictedly rectangularly convergent at almost every point but which is triangularly divergent everywhere.

Consider the series

$$
b(x, y)=\sum_{l=1}^{\infty} 2^{l} \exp \left(i 4^{l} x\right) \sin ^{2 l} y=\sum_{m, n=-l}^{\infty} b_{m, n} e^{i(m x+n y)} .
$$

For each fixed $l$, the series contributes terms with indices

$$
\left(4^{l},-2^{l}\right), \quad\left(4^{l},-2^{l}+2\right), \quad\left(4^{l},-2^{l}+4\right), \quad \ldots, \quad\left(4^{l}, 2^{l}\right) .
$$

In the lattice plane (see Figure 2) all of these points are contained within the set $H=\left\{(m, n)|| m \mid \geqq n^{2}\right\}$. Let an eccentricity $e>1$ be specified. Since if $l$ is sufficiently large all of the points of (3.24) lie outside of the region $R=\left\{(m, n)\left|e^{-1} \leqq\right| m|n| \leqq e\right\}$ (precisely, when $4^{l} / e>\left(4^{l}\right)^{1 / 2}$ ), it follows that the restrictedly rectangular partial sums of eccentricity bounded by $e$ eventually coincide with the ordinary partial sums of $b(x, y)$. These, in turn, converge almost everywhere for if $|\sin y|<1$, $b(x, y)$ is majorized by

$$
\sum_{l=1}^{\infty} 2^{l}|\sin y|^{2^{l}}<\sum_{n=1}^{\infty} n|\sin y|^{n}<\infty
$$

and hence converges absolutely. Hence, $b(x, y)$ converges restrictedly rectangularly almost everywhere.

However, if we let $t(R)$ denote the triangular partial sum given by

$$
t(R)=\sum_{|m|+|n| \leqq R} b_{m, n} \exp \left(i\left(m x_{0}+n y_{0}\right)\right)
$$

where $\left(x_{0}, y_{0}\right)$ is any fixed point, then if $b$ converges triangularly at $\left(x_{0}, y_{0}\right)$, in particular

$$
t(R+1)-t(R) \rightarrow 0
$$

as $R \rightarrow \infty$. But $t\left(4^{l}-1\right)$ is equal to the $\left(4^{l}-1\right)$ th partial sum of $b\left(x_{0}, y_{0}\right)$ (since $4^{l-1}+2^{l-1}<4^{l}-1, l=1,2,3, \ldots$-see Figure 2) whereas $t\left(4^{l}\right)$ contains the same terms together with $b_{4^{l}, 0} \exp \left(i 4^{l} x\right)$ so that (see (2.22))

$$
\left|t\left(4^{l}\right)-t\left(4^{l}-1\right)\right|=\left|b_{4^{l}, 0}\right|=\left|2^{l} 2^{-2^{l}}\left(\begin{array}{c}
2^{l} \\
2^{l-1}
\end{array}\right)\right|>\frac{2^{l / 2}}{3}
$$

which contradicts (3.26).

EXAMPLE 3.3. There is a series which is restrictedly rectangularly convergent at almost every point but which is circularly divergent everywhere.

The same example $b(x, y)$ mentioned above (see (3.23)) also is circularly divergent. The proof is similar to that of triangular divergence given in Example 3.2. Fix $\left(x_{0}, y_{0}\right)$ and let

$$
S(R)=\sum_{|m|+|n| \leqq R} b_{m, n} \exp \left(i\left(m x_{0}+n y_{0}\right)\right) .
$$




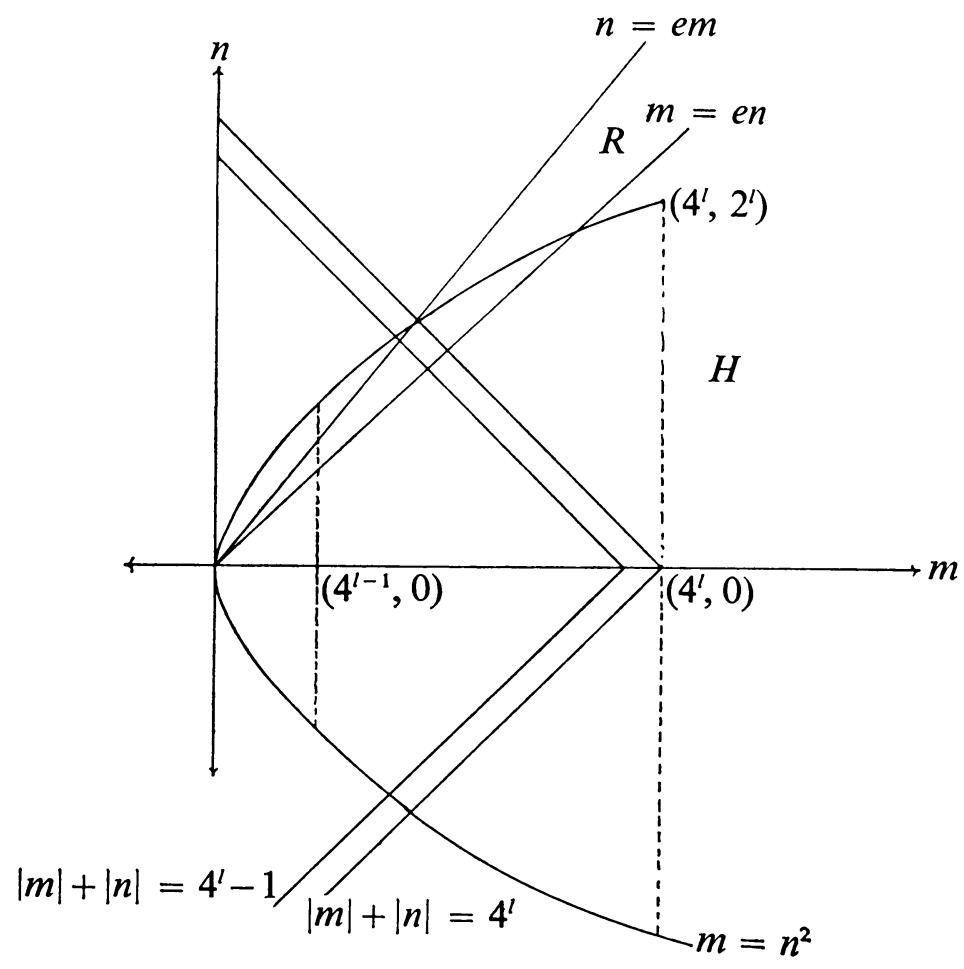

FIGURE 2

As above, to see that $\lim _{R \rightarrow \infty} S(R)$ does not exist it suffices to show that

$$
S\left(4^{2 l}\right)-S\left(4^{2 l}-1\right)=b_{4^{l}, 0} \exp \left(i 4^{l} x\right) \text {. }
$$

Here, we wish to point out that Example 2.1 is even stronger than Example 3.3 since the theorems of R. Cooke [3] and A. Zygmund [25] show (2.33) can converge circularly only in a set of zero measure since its coefficients do not tend to zero. Example 2.1 is a stronger example since it not only converges restrictedly but also converges unrestrictedly almost everywhere. The advantage of Example 3.3 is that it is easy to see that it is circularly divergent everywhere while the set of circular divergence of Example 2.1 is not known to us.

EXAMPLE 3.4. In Example 3.2, one saw that the square partial sums of $b(x, y)$ converge almost everywhere and that the coefficients of $b(x, y)$ do not tend to zero. By rotating the torus with the change of variables $x=x^{\prime}+y^{\prime}$ and $y=x^{\prime}-y^{\prime}$ we obtain a series $c\left(x^{\prime}, y^{\prime}\right)$ which is triangularly convergent almost everywhere. However, since the coefficients do not tend to zero, $c\left(x^{\prime}, y^{\prime}\right)$ does not converge circularly in a set of positive measure by the Cooke-Zygmund result. Furthermore, the reasoning used in Example 3.2 shows that $c\left(x^{\prime}, y^{\prime}\right)$ is nowhere convergent for square partial sums. 
EXAMPLE 3.5. There is a series which is convergent at almost every point but which is triangularly divergent almost everywhere.

To see this we shall define a series $L(x, y)=M(x) \delta(y)$ of the type considered in Example 2.1. As before, $\delta(y)=1+2 \sum_{n=1}^{\infty} \cos n y$. We choose $M(x)=\sum_{n=1}^{\infty} b_{n} \sin n x$ to be the Fourier-Stieltjes series of a Lebesgue function $F(x)$. The function $F(x)$ is defined by $F(x)=\lim _{p \rightarrow \infty} F_{p}(x)$ for $-\pi \leqq x \leqq \pi$ where the continuous function $F_{p}(x)$ satisfies $F_{p}(0)=0, F_{p}(\pi)=\pi / 2 ; F_{p}$ increases linearly by $2^{-p}(\pi / 2)$ on each of the $2^{p}$ intervals of length $(2 / 5)^{p}$ with left-hand endpoint $\alpha_{0}+\alpha_{1}\left(\frac{2}{5}\right)+\cdots+\alpha_{p}\left(\frac{2}{5}\right)^{p-1}$ where each $\alpha_{j}$ is 0 or $\left(\frac{3}{5}\right) \pi ; F_{p}(x)$ is constant elsewhere on $[0, \pi]$; and $F_{p}$ is extended to $[-\pi, 0]$ by $F_{p}(x)=F_{p}(-x)$. A calculation similar to the one in [23, Vol. I, p. 195] shows that $b_{n}=0$ if $n$ is even and

$$
b_{n}=(-1)^{(n-1) / 2} \prod_{k=0}^{\infty} \cos \left(\frac{3}{10} \pi n\left(\frac{2}{5}\right)^{k}\right)
$$

if $n$ is odd so that in any case $\left|b_{n}\right| \leqq\left|\gamma\left(\frac{3}{10} n\right)\right|$ where $\gamma(u)=\prod_{k=0}^{\infty} \cos \left(\pi u\left(\frac{2}{5}\right)^{k}\right)$, so that $b_{n} \rightarrow 0$ [23, Vol. II, pp. 147-148, and Theorem 11.16 on p. 151]. It follows that $M(x)$ converges to zero almost everywhere but not everywhere [23, Vol. I, pp. 347-348, especially the sufficiency part of Theorem 6.8 and the statement of Theorem 6.11]. The conjugate series $\tilde{M}(x)=-\sum_{n=1}^{\infty} b_{n} \cos n x$ converges almost everywhere [23, Vol. II, p. 216, Theorem 4.1] and for every $y,|\{x \mid \tilde{M}(x)=y\}|=0$. This is so for otherwise the function $u(z)=u\left(r e^{i x}\right)=\sum_{n=1}^{\infty} b_{n}\left(r e^{i x}\right)^{n}$ which is analytic on $|z|<1$ (since $b_{n} \rightarrow 0$ ) would have a constant nontangential limit on a subset of $|z|=1$ of positive linear measure [23, Vol. I, p. 100 , Theorem $7.6 ;$ p. 105 , $\S 8$, first two sentences; and p. 253, Theorem 1.6] which would force all $b_{n}$ to be zero [23, Vol. II, p. 203, Theorem 1.9] which is impossible since $M(x)$ does not converge to zero everywhere.

By the argument used in Example 2.1 it follows that $L(x, y)$ is convergent almost everywhere. To show that $L(x, y)$ is triangularly divergent almost everywhere, it suffices to show that

$$
\Delta_{N}(x, y)=t_{N}(x, y)-t_{N-1}(x, y)=\sum_{|m|+|n|=N} l_{m, n} e^{i(m x+n y)}
$$

does not tend to zero at almost every $(x, y) \in T^{2}$. Using several elementary trigonometric identities we find that

$$
\begin{aligned}
\Delta_{N}(x, y)= & \sum_{m=1}^{N-1} b_{m} \sin m x(2 \cos (N-m) y)+b_{N} \sin N x \\
= & 2 \sum_{m=1}^{N-1} b_{m} \sin m x(\cos N y \cos m y+\sin N y \sin m y)+o(1) \\
= & \cos N y \sum_{m=1}^{N-1} b_{m}\{\sin m(x-y)+\sin m(x+y)\} \\
& +\sin N y \sum_{m=1}^{N-1} b_{m}\{\cos m(x-y)-\cos m(x+y)\}+o(1) .
\end{aligned}
$$


For almost every $(x, y) \in T^{2}, M(x-y)=M(x+y)=0$ and $\tilde{M}(x-y)$ and $\tilde{M}(x+y)$ exist. For such an $(x, y)$, we have

$$
\begin{aligned}
\Delta_{N}(x, y) & =o(1)+\sin N y\{\tilde{M}(x+y)-\tilde{M}(x-y)+o(1)\}+o(1) \\
& =\sin N y\{\tilde{M}(x+y)-\tilde{M}(x-y)\}+o(1) .
\end{aligned}
$$

Since $\tilde{M}$ is not constant on any set of positive measure, the quantity in braces is not zero almost everywhere. Since for almost every $y, \sin N y$ does not tend to zero [23, Vol. I, p. 142, Corollary to Theorem 4.27], neither does $\Delta_{N}(x, y)$ as was to be shown.

This example is stronger than Example 3.2, but is somewhat less constructive since here the sets of convergence and divergence are not specified.

REMARKS AND PROBLEMS. It should be mentioned explicitly that the above examples show most common regular methods are pairwise inequivalent, even on an almost everywhere basis. For example, $b(x, y)$ is almost everywhere square convergent but is nowhere circularly convergent.

We do not know of examples of series which are circularly convergent but not square (or, equivalently, triangularly) convergent on a set of positive measure. The situation may be summarized by the following diagram in which $A H B$ means that there is a trigonometric series convergent almost everywhere with respect to method $A$ but convergent on no set of positive measure with respect to method $B$, while $C \rightarrow D$ means that convergence with respect to method $C$ on a set forces convergence with respect to method $D$ almost everywhere on that set.

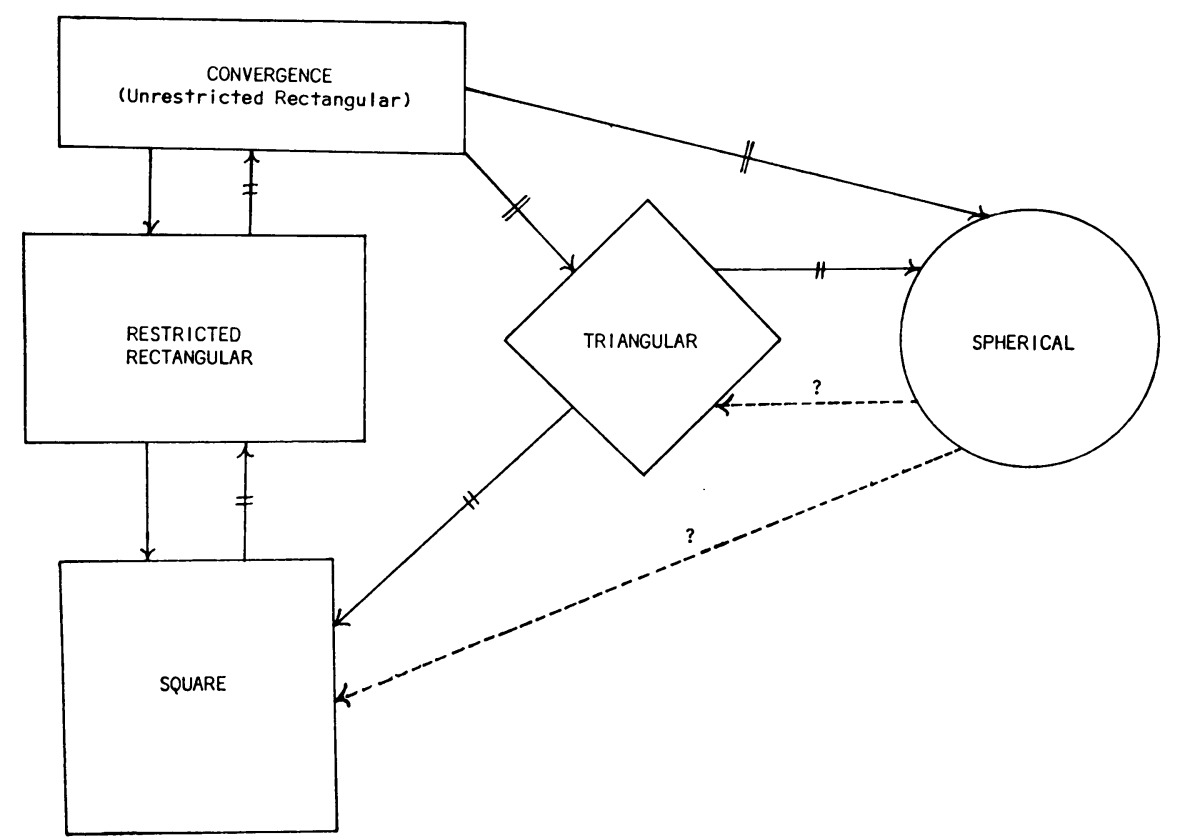

Figure 3 
Despite the basic incompatibility between various methods of convergence, if one sticks to a given method, consistency theorems of the form "convergence implies summability" are often true. For example, if the numerical series

$$
\sum_{i=0}^{\infty} \sum_{j=0}^{\infty} a_{i j}
$$

is square convergent to $s$, then the $(C, 1)$ means

$$
\sigma_{n, n}=\frac{1}{n+1} \sum_{i=0}^{n} s_{i i}
$$

where $s_{m, n}=\sum_{i=0}^{m} \sum_{j=0}^{n} a_{i j}$, also converge to $s$. Similar theorems hold for spherical and triangular partial sums for here also the situation is essentially one-dimensional. However, one sometimes needs the additional assumption of boundedness of the partial sums for consistency. For example, the numerical series $\sum b_{i}$, where $b_{0 j}=1$, $j=1,2, \ldots, b_{i i}=-1, i=1,2, \ldots, b_{i j}=0$ otherwise is square convergent to 0 since all $s_{n, n}=0$ but has $(C, 1,1)$ means

$$
\sigma_{n, n}=\frac{1}{(n+1)^{2}} \sum_{i=0}^{n} \sum_{j=0}^{n} s_{i j}=\frac{n(n+2)}{6(n+1)}
$$

and so is not $(C, 1,1)$ square summable. This is the motivation behind saying that a series converges in the sense of Pringsheim if it is unrestrictedly rectangularly convergent and has bounded partial sums. A typical consistency theorem is

LEMma 3.3. If the numerical series (3.30) converges in the sense of Pringsheim to $s$, then it is summable $(C, 1,1)$ to $s$, that is,

$$
\lim _{\min \{m, n\} \rightarrow \infty} \frac{\sigma_{m, n}}{(m+1)(n+1)}=\lim _{\min \{m, n\} \rightarrow \infty} \frac{1}{(m+1)(n+1)} \sum_{i=0}^{m} \sum_{j=0}^{n} s_{i j}=s .
$$

Proof. Given $\varepsilon>0$ pick $\mu$ so large that $\left|s_{i j}-s\right|<\varepsilon / 3$ if $i$ and $j$ are $\geqq \mu$. Then pick $n$ and $m$ so large that $\mu B /(m+1)<\varepsilon / 3$ and $\mu B /(n+1)<\varepsilon / 3$ where $B=\sup _{i, j}\left|s_{i j}-s\right|$ is finite by hypothesis. Then

$$
\begin{aligned}
& \left|\frac{\sigma_{m, n}}{(m+1)(n+1)}-s\right|=\left|\frac{1}{(m+1)(n+1)} \sum_{i=0}^{m} \sum_{j=0}^{n}\left(s_{i j}-s\right)\right| \\
& \quad \leqq \frac{1}{(m+1)(n+1)} \sum_{i=0}^{\mu-1} \sum_{j=0}^{n} B+\frac{1}{(m+1)(n+1)} \sum_{i=0}^{m} \sum_{j=0}^{\mu-1} B+\frac{1}{(m+1)(n+1)} \sum_{i=\mu}^{m} \sum_{j=\mu}^{n} \frac{\varepsilon}{3} \\
& \quad<\frac{\varepsilon}{3}+\frac{\varepsilon}{3}+\frac{\varepsilon}{3}=\varepsilon .
\end{aligned}
$$

From Lemmas 2.3 and 3.3 we deduce

THEOREM 3.2. If a trigonometric series converges on a set $E$ of positive measure, then the series is $(C, 1,1)$ summable at almost every point of $E$. 
Theorem 3.2 differs from the already known Lemma 3.3 only in that the hypothesis of boundedness of the partial sums has been removed. There are many similar classical theorems in which the hypothesis of bounded partial sums may be dropped. It would be repetitious to list any more of them.

IV. Uniqueness of multiple trigonometric series. The problem of uniqueness for spherical summability has been discussed by Victor Shapiro in [16], where he proved

TheOrem 4.1 (ShaPIRo). Given the multiple trigonometric series

$$
\sum a_{m} e^{i m \cdot x}
$$

where the $a_{m}$ are arbitrary complex numbers. Let $q$ be a point on $T^{k}$. Suppose that

(4.2a) $\sum_{R-1<|m| \leqq R}\left|a_{m}\right|=o(R)$ as $R \rightarrow \infty$,

(4.2b) $f^{*}(\boldsymbol{x})$ and $f_{*}(\boldsymbol{x})$ are finite for $\boldsymbol{x}$ in $T^{k}-\{\boldsymbol{q}\}$,

(4.2c) $f^{*}$ is in $L^{1}$ on $T^{k}$,

(4.2d) $f_{*}$ is in $L^{1}$ on $T^{k}$,

where $f_{*}(x)$ is the lower Abel sum of the series (4.1) given by

$$
f_{*}(x)=\liminf _{h \rightarrow 0} \sum a_{m} e^{i m \cdot x-|m| h}
$$

and $f^{*}(x)$, the upper Abel sum, is analogously defined. Then the series (4.1) is the Fourier series of $f_{*}(\boldsymbol{x})$.

A partial uniqueness theorem for triangular partial sums has been obtained by George Cross [4]. M. H. Nasibov [12] has proved uniqueness theorems for unrestricted and restricted convergence, but his hypotheses are rather strong. All of these theorems assume something about the rate of growth of the coefficients. The following theorem avoids this problem, but we have been able to prove it only in two dimensions.

THEOREM 4.2. Let the trigonometric series

$$
\sum a_{m, n} e^{i(m x+n y)}
$$

be convergent everywhere on $T^{2}$ to the finite-valued Lebesgue integrable function $f(x, y)$. Then (4.3) is the Fourier series of $f$.

Recall that convergent means unrestrictedly rectangularly convergent. Let $f_{0}(x, y)$ be defined by

$$
f_{0}(x, y)=\liminf _{\min \{|m|,|n|\} \rightarrow \infty} \sum a_{m, n} e^{\{(m x+n y)}
$$

and $f^{\circ}(x, y)$ be defined analogously. Let $q=\left(q_{1}, q_{2}\right)$ be any point of $T^{2}$. Then a somewhat more general version of Theorem 4.2 is 
THEOREM 4.2'. Suppose that

(4.4a) $f^{0}(x, y)$ and $f_{0}(x, y)$ are finite for $(x, y)$ in $T^{2}-\{\boldsymbol{q}\}$,

(4.4b) $f^{0}$ is in $L^{1}$ on $T^{2}$,

(4.4c) $f_{0}$ is in $L^{1}$ on $T^{2}$.

Then the series (4.3) is the Fourier series of $f_{0}$.

COROLlary 4.1. Suppose that the trigonometric series (4.3) converges to zero on $T^{2}$. Then the series is identically zero, that is, all the coefficients are zero.

The corollary is obviously Theorem 4.2 in the special case when $f(x, y) \equiv 0$. We proceed now to the proof of Theorem 4.2. The proof is an application of Shapiro's theorem. From Lemma 2.3 and Lemma 3.1 it is clear that conditions (4.2b, c, and d) are satisfied. To see that condition (4.2a) holds, by virtue of Theorem 2.2 , we need only prove

LEMMA 4.1. If

$$
\left|a_{m, n}\right| \leqq C
$$

and

then

$$
\lim _{:(m, n) \| \rightarrow \infty} a_{m, n}=0
$$

$$
\sum_{R-1<|(m, n)| \leqq R}\left|a_{m, n}\right|=o(R) \quad \text { as } R \rightarrow \infty .
$$

Proof. This is geometrically evident. For we write

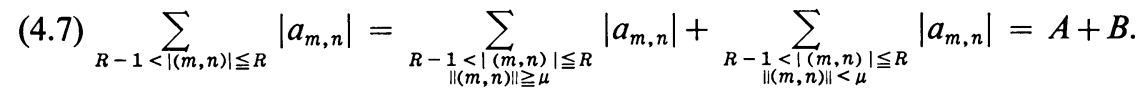

If $\mu$ is chosen sufficiently large each term of $A$ will be arbitrarily small by (4.6). Since there are (asymptotically) only $2 \pi R$ terms altogether (see Rademacher [13, p. 100]), it follows that $A$ is $o(R)$ as $R \rightarrow \infty$. Once $\mu$ is fixed, $B$ is seen to have $o(R)$ terms as $R$ increases so that, by (4.5), $B$ also is $o(R)$ as $R \rightarrow \infty$. This finishes Lemma 4.1 and, hence, Theorem 4.2. The proof of Theorem $4.2^{\prime}$ uses the full strength of Shapiro's theorem and is similar to the proof just given. Instead of Lemma 3.1, one uses the fact that

$$
f_{0}(\boldsymbol{x}) \leqq f_{*}(\boldsymbol{x}) \leqq f^{*}(\boldsymbol{x}) \leqq f^{0}(\boldsymbol{x}) .
$$

This last fact follows from Lemma 2.3, the fact that $\lambda_{m}(h)=e^{-|m| h}$ satisfies the hypotheses of Lemma 3.2, and an argument very close to the one which proves Lemma 3.2.

If the dimension of the space is at least three, then we can prove only the following partial result.

THEOREM 4.3. If, for every $x$ in a set of positive measure,

$$
\sum_{n \geqq 0} a_{n} e^{i n \cdot x}=0,
$$

then all $a_{n}$ are zero. 
In order to prove this theorem we first need to establish the consistency of nontangential Abel summability.

Let $z=\left(z_{1}, z_{2}, \ldots, z_{k}\right), z^{0}=\left(z_{1}^{0}, \ldots, z_{k}^{0}\right)$ where each $z_{j}$ is a complex number of modulus less than one and $z^{0}$ is a point of $T^{k}$, so that $z_{j}^{0}=\exp \left(i x_{j}\right)$ for each $j$. We say that $z$ tends nontangentially to $z^{0}$ if each $z_{j}$ tends nontangentially to $z_{j}^{0}$. In other words there is a constant $M$ so that

$$
\left|z_{j}^{0}-z_{j}\right| /\left(1-\left|z_{j}\right|\right) \leqq M, \quad j=1, \ldots, k .
$$

Note that $M$ is necessarily bigger than or equal to one.

If $\phi(z)=\sum_{n \geqq 0} a_{n} z^{n}$ converges whenever all $\left|z_{j}\right|<1$ (here $z^{n}=z_{1}^{n}{ }_{1} z_{2}^{n} \cdots z_{k}^{n}$ ) and if $\phi(z)$ tends to $s$ as $z$ tends nontangentially to $z^{0}$, then we say that the series $\sum a_{n}\left(z^{0}\right)^{n}$ $=\sum a_{n} e^{i n \cdot x}$ is summable $A^{*}$ to $s$.

LEMMA 4.2. If

and

$$
\Sigma=\sum_{n \geqq 0} a_{n} e^{i n \cdot x}=s \quad \text { unrestricted rectangularly, }
$$

$$
\left|\sum_{N \geqq n \geqq 0} a_{n} e^{i n \cdot x}\right| \leqq B \text { for all } N
$$

then $\Sigma$ is summable $A^{*}$ to $s$.

Proof. An application of Abel's summation by parts formula (3.3) shows that it is sufficient to prove that

$$
\sum_{(p)}\left|\Delta^{p}\left(\boldsymbol{w}^{m}\right)\right| \leqq M^{k}
$$

for each $p \in \Omega^{k}$, where $M$ is given by (4.10);

$$
\underset{z \rightarrow z^{0} \text { nontang }}{\operatorname{limit}} \sum_{(p)}^{\prime}\left|\Delta^{p}\left(\boldsymbol{w}^{\boldsymbol{m}}\right)\right|=0
$$

for each fixed $p \in \Omega^{k}$, fixed choice of omitted summation, and fixed set of $m_{j}$ 's corresponding to the indices not summed; and

$$
w^{0}=1,
$$

where $\boldsymbol{w}=\left(z_{1}\left(z_{1}^{0}\right)^{-1}, \ldots, z_{k}\left(z_{k}^{0}\right)^{-1}\right)$.

Refer to Lemmas 3.1 and 3.2 for the notation. The sufficiency of conditions (4.11)-(4.13) is so similar to the proof of Lemma 3.2 that we omit it. Condition (4.13) holds by definition. To ease notation let $Q=(1, \ldots, 1,0, \ldots, 0)$ have its first $r$ entries equal to 1 and its remaining $k-r$ entries equal to 0 . We must show

$$
\sum_{n_{1}=0}^{\infty} \cdots \sum_{n_{r}=0}^{\infty}\left|\Delta^{Q}(w)^{\left(n_{1}, \ldots, n_{r}, N_{r+1}, \ldots, N_{k}\right)}\right| \leqq M^{k}
$$

and, say, that

$$
\sum_{n_{1}=0}^{\infty} \ldots \sum_{n_{r}-1=0}^{\infty}\left|\Delta^{Q}(\boldsymbol{w})^{\left(n_{1}, \ldots, n_{r}, N_{r+1}, \ldots, N_{k}\right)}\right| \rightarrow 0
$$


as $\boldsymbol{w} \rightarrow \mathbf{1}$ nontangentially for each fixed choice of $n_{r}, N_{r+1}, \ldots$, and $N_{k}$. (Note that $\left|1-w_{j}\right| /\left(1-\left|w_{j}\right|\right)=\left|z_{j}^{0}-z_{j}\right| /\left(1-\left|z_{j}\right|\right)$.)

Now

$$
\begin{aligned}
\left|\Delta^{Q}(\boldsymbol{w})^{\left(n_{1}, \cdots, N_{k}\right)}\right| & =\left|\left(w_{1}^{n_{1}}-w_{1}^{n_{1}+1}\right) \cdots\left(w_{r}^{n_{r}}-w_{r}^{n_{r}+1}\right) w_{r+1}^{N_{r}+1} \cdots w_{k}^{N}\right| \\
& =\left|w_{1}\right|^{n_{1}} \cdots\left|w_{k}\right|^{N_{k}}\left|1-w_{1}\right|\left|1-w_{2}\right| \cdots\left|1-w_{r}\right| \\
& \leqq \prod_{j=1}^{r}\left|1-w_{j}\right| \cdot\left|w_{j}\right|^{n_{j}}
\end{aligned}
$$

so that the left-hand side of (4.14) is dominated by

$$
\prod_{j=1}^{r}\left(\left|1-w_{j}\right| \sum_{n_{j}=0}^{\infty}\left|w_{j}\right|^{n_{j}}\right)=\prod_{j=1}^{r}\left(\left|1-w_{j}\right| /\left(1-\left|w_{j}\right|\right)\right) \leqq M^{r} \leqq M^{k}
$$

while the left-hand side of (4.15) is dominated by

$$
\left|1-w_{r}\right| \prod_{j=1}^{r-1}\left(\left|1-w_{j}\right| /\left(1-\left|w_{j}\right|\right)\right) \leqq\left|1-w_{r}\right| M^{k-1}
$$

which tends to zero as $\boldsymbol{w} \rightarrow \mathbf{1}$, completing the proof of Lemma 4.2.

Assume now that (4.9) holds on a set $E$ of positive measure. By Lemma 2.3 there is a set $F \subset E$ of full measure on which the partial sums are bounded. The function

$$
\phi(z)=\sum_{n \geqq 0} a_{n} z^{n}
$$

is absolutely convergent in $\left\{z:\left|z_{1}\right|<1, \ldots,\left|z_{k}\right|<1\right\}$ since the coefficients are bounded by Theorem 2.2. Furthermore, by Lemma 4.2, $\phi(z)$ has nontangential limit 0 at each point of $F$, and $|F|>0$. These two facts are exactly the hypotheses of a theorem of Calderón whose conclusion is that $a_{n}=0$ for every $n$ [23, Vol. II, p. 321, Theorem 4.24].

REMARKS. It is an open question whether or not a uniqueness theorem holds for general series in dimensions greater than two. In dimension three Shapiro's theorem cannot be used because the analogue of Lemma 4.1 does not go through. In fact, one may easily find numbers $a_{l m n}$ such that $a_{l m n} \rightarrow 0$ as $\|(l, m, n)\| \rightarrow \infty$ but

$$
\sum_{R-1<\mid(l, m, n) ! \leqq R}\left|a_{l m n}\right| \neq O(R),
$$

for example, $a_{l m n}=\left(l^{2}+m^{2}+n^{2}\right)^{-1 / 4}$. Theorem 4.3 has little bearing on the question of uniqueness for general trigonometric series. For example, if $k=1$, the series $\sum n \sin n x$ is summable $A^{*}$ to zero at all points except $x=0$ (and, hence, $a$ fortiori on a set of positive measure). This helps to illustrate the fact that the question of uniqueness is much more delicate for general trigonometric series than for trigonometric series of power series type.

A different approach to the question of uniqueness may be made by considering the $2 k$ th integral. For simplicity let $k=2$ and $a_{0, n}=a_{m, 0}=0$. Assume that for every $(x, y)$ in $T^{2}$

$$
\sum_{m=-\infty}^{\infty} \sum_{n=-\infty}^{\infty} a_{m, n} e^{i(m x+n y)}=0
$$


unrestrictedly rectangularly. Form the continuous function

$$
F(x, y)=\sum_{m=-\infty}^{\infty} \sum_{n=-\infty}^{\infty} \frac{a_{m, n}}{m^{2} n^{2}} e^{i(m x+n y)} .
$$

We have the following consistency theorem.

THEOREM 4.4. (See Zak [22].) If we assume (4.16) and set

$$
\begin{aligned}
\Delta^{(2,2)} F(x, y ; h, k)= & F(x+h, y+k)-2 F(x+h, y)+F(x+h, y-k) \\
& -2 F(x, y+k)+4 F(x, y)-2 F(x, y-k) \\
& +F(x-h, y+k)-2 F(x-h, y)+F(x-h, y-k)
\end{aligned}
$$

then for all $(x, y)$ in $T^{2}$

$$
D^{(2,2)} F(x, y)=\lim _{h, k \rightarrow 0} \frac{\Delta^{(2,2)} F(x, y ; h, k)}{h^{2} k^{2}}=0 \quad(h, k \neq 0)
$$

where the limit is taken as $h$ and $k$ tend to zero jointly but with unrestricted ratio.

Proof. Using Abel's partial summation formula (3.3) and observing that $\left|s_{m, n}(x, y)\right| \leqq B(x, y)$ (Lemma 2.3) we compute

$$
\begin{aligned}
\frac{\Delta^{(2,2)} F(x, y ; h, k)}{h^{2} k^{2}} & =\sum_{m=-\infty}^{\infty} \sum_{n=-\infty}^{\infty} a_{m, n} e^{i(m x+n y)}\left(\frac{\sin m h}{m h}\right)^{2}\left(\frac{\sin n k}{n k}\right)^{2} \\
& =\sum_{m=-\infty}^{\infty} \sum_{n=-\infty}^{\infty} s_{m, n}(x, y) \lambda_{m, n}(h, k)
\end{aligned}
$$

where

(4.21) $\lambda_{m, n}(h, k)=\left[\left(\frac{\sin m h}{m h}\right)^{2}-\left(\frac{\sin (m+1) h}{(m+1) h}\right)^{2}\right] \cdot\left[\left(\frac{\sin n k}{n k}\right)^{2}-\left(\frac{\sin (n+1) k}{(n+1) k}\right)^{2}\right]$.

Decompose (4.20) into four terms.

$$
\begin{aligned}
\sum_{m=-\infty}^{\infty} \sum_{n=-\infty}^{\infty} s_{m, n} \lambda_{m, n} & =\sum_{m=-\mu}^{\mu} \sum_{n=-\mu}^{\mu}+\sum_{m=-\mu}^{\mu} \sum_{|n|>\mu}+\sum_{|m|>\mu} \sum_{n=-\mu}^{\mu}+\sum_{|m|>\mu} \sum_{|n|>\mu} \\
& =A+B+D+D+D+D+D
\end{aligned}
$$

If $\mu$ is large, $s_{m, n}$ is small for $m$ and $n>\mu$ so $D$ is small since

$$
\sum_{m=-\infty}^{\infty} \sum_{n=-\infty}^{\infty}\left|\lambda_{m, n}(h, k)\right| \leqq \int_{-\infty}^{\infty} \int_{-\infty}^{\infty}\left|\left(\frac{\sin u}{u}\right)^{2 \prime}\left(\frac{\sin v}{v}\right)^{2 \prime}\right| d u d v<\infty
$$

For the remaining terms note that $\left|s_{m, n}(x, y)\right| \leqq B(x, y)$ by Lemma 2.3. Then $A$ can be made small by choosing $h$ and $k$ small since, for each $m$ and $n$,

$$
\lim _{h, k \rightarrow 0} \lambda_{m, n}(h, k)=0 .
$$

Similarly, if $h$ and $k$ are small $B$ is small since for each $m$

$$
\begin{aligned}
\lim _{h, k \rightarrow 0} & \sum_{n=-\infty}^{\infty}\left|\lambda_{m, n}(h, k)\right| \\
& \leqq \lim _{h, k \rightarrow 0} \int_{-\infty}^{\infty}\left|\left(\frac{\sin v}{v}\right)^{2 \prime}\right| d v \cdot\left|\left(\frac{\sin m h}{m h}\right)^{2}-\left(\frac{\sin (m+1) h}{(m+1) h}\right)^{2}\right|=0
\end{aligned}
$$


while a symmetrical argument shows $C$ small.

Hence, (4.22) tends to zero with $h$ and $k$, which proves Theorem 4.4 (see Robison $[15$, p. 67]).

By virtue of Theorem 4.4, one would have a different proof of Theorem 4.2 which would probably generalize to higher dimensions if one could prove

Conjecture 1. Let $F$ be continuous on $T^{2}$. If

$$
D^{(2,2)} F=0 \text { for all }(x, y) \text { in } T^{2} \text {, }
$$

then there are functions $a(y), b(y), c(x)$, and $d(x)$ such that

$$
F(x, y)=a x+b+c y+d .
$$

Comments. This would generalize Theorem 10.7 on p. 23 of [23, Vol. I]. Without loss of generality we may add the hypothesis that

$$
F=0 \text { on the boundary of }[-\pi, \pi] \times[-\pi, \pi]
$$

and then attempt to deduce that $F$ is identically zero on $T^{2}$ since $F(x, y)$ may be replaced by

$G(x, y)=F(x, y)-\left\{\frac{1}{2 \pi}[F(x, \pi)-F(x,-\pi)]\right.$

$$
\begin{gathered}
+\frac{x}{4 \pi^{2}}[F(\pi,-\pi)+F(-\pi, \pi)-F(\pi, \pi)-F(-\pi,-\pi)] \\
\left.+\frac{1}{4 \pi}[F(\pi,-\pi)+F(-\pi,-\pi)-F(\pi, \pi)-F(-\pi, \pi)]\right\} y \\
-\left\{\frac{1}{2}[F(x, \pi)+F(x,-\pi)]+\frac{x}{4 \pi}[F(-\pi, \pi)+F(-\pi,-\pi)-F(\pi, \pi)-F(\pi,-\pi)]\right. \\
\left.-\frac{1}{4}[F(\pi, \pi)+F(-\pi, \pi)+F(\pi,-\pi)+F(-\pi,-\pi)]\right\} \\
-\left\{\frac{1}{2 \pi}[F(\pi, y)-F(-\pi, y)]\right\} x-\left\{\frac{1}{2}[F(-\pi, y)+F(\pi, y)]\right\}
\end{gathered}
$$

where $G(x, y)$ differs from $F(x, y)$ by a function of the form (4.27) and $G(\pi, y)$ $=G(-\pi, y)=G(x, \pi)=G(x,-\pi)=0$ as may be verified directly. We now observe

LEMMA 4.3. Let $F(x, y)$ be continuous on $T^{2}$. If

$$
F(x, y)=0 \text { on the boundary of }[-\pi, \pi] \times[-\pi, \pi]
$$

and

$$
\Delta^{(2,2)} F(x, y ; h, k) \equiv 0
$$

then $F(x, y) \equiv 0$.

Proof. Setting $x=y=0, h=k=\pi$ and applying (4.18), it follows from (4.30) and (4.31) that $F(0,0)=0$. Setting $(x, y ; h, k)=(0, \pi / 2 ; \pi, \pi / 2)$ and reasoning similarly shows that $F(0 ; \pi / 2)=0$. From considering $(x, y ; h, k)=(-\pi / 2,0 ; \pi / 2, \pi)$ 
we get $F(-\pi / 2,0)=0$. Proceeding inductively shows that $F$ is zero on the countable dense set

$$
\begin{aligned}
\left\{(0,0) ;\left(0, \frac{\pi}{2}\right),\left(-\frac{\pi}{2}, 0\right),\left(0,-\frac{\pi}{2}\right),\left(\frac{\pi}{2}, 0\right) ;\right. \\
\left.\quad\left(\frac{\pi}{2}, \frac{\pi}{2}\right),\left(-\frac{\pi}{2}, \frac{\pi}{2}\right),\left(-\frac{\pi}{2},-\frac{\pi}{2}\right),\left(\frac{\pi}{2},-\frac{\pi}{2}\right) ;\left(0, \frac{\pi}{4}\right), \ldots\right\} .
\end{aligned}
$$

(For example, setting $(x, y ; h, k)=(0, \pi / 4 ; \pi, \pi / 8)$ and using $F(0,0)=F(0, \pi / 2)=0$, we find that $F(0, \pi / 4)=0$.) It then follows by continuity that $F$ is identically zero. Because of Lemma 4.3, in order to prove Conjecture 1 it would suffice to prove the following mean value type statement.

ConjeCture 2. If $F$ is continuous on $T^{2}$ and $D^{(2,2)} F \equiv 0$ on $T^{2}$, then

$$
\Delta^{(2,2)} F(x, y ; h, k) \equiv 0 .
$$

Conjectures 1 and 2 and Lemma 4.3 were stated in [8, esp. pp. 95-108], but without sufficient proof.

Another approach to the question of uniqueness which yields only partial results may be made as follows. For simplicity we let $k=2, a_{0,0}=0$ and consider the trigonometric series

$$
t=\sum a_{n} e^{i n \cdot x}=\sum_{m=-\infty}^{\infty} \sum_{n=-\infty}^{\infty} a_{m, n} e^{i(m x+n y)} .
$$

Define (see Shapiro, [17])

$$
t^{(1)}=\left(-\sum a_{n} e^{i n \cdot x} \frac{i m}{|\boldsymbol{n}|^{2}},-\sum a_{n} e^{i n \cdot x} \frac{i n}{|\boldsymbol{n}|^{2}}\right)=\left(t_{1}^{(1)}, t_{2}^{(1)}\right)
$$

Then $t^{(1)}$ is a formal integral of $t$ in the sense that

$$
\operatorname{grad} t^{(1)}=\partial t_{1}^{(1)} / \partial x+\partial t_{2}^{(1)} / \partial y=t
$$

where the partial derivatives are computed termwise and the last equality is formal.

Repeat the process forming $t^{(2)}=\left(t^{(1)}\right)^{(1)}$,

$$
t^{(2)}=\left(\begin{array}{ll}
-\sum a_{n} e^{i n \cdot x} m^{2} /|\boldsymbol{n}|^{4} & -\sum a_{n} e^{i n \cdot x} m n /|n|^{4} \\
-\sum a_{n} e^{i n \cdot x} m n /|\boldsymbol{n}|^{4} & -\sum a_{n} e^{i n \cdot x} n^{2} /|\boldsymbol{n}|^{4}
\end{array}\right)=\left(\begin{array}{cc}
t_{11}^{(2)} & t_{12}^{(2)} \\
t_{21}^{(2)} & t_{22}^{(2)}
\end{array}\right)
$$

We find that $t^{(2)}$ is a formal second integral of $t$ in the sense that

$$
\operatorname{grad} \circ \operatorname{grad} t^{(2)}=\frac{\partial^{2}}{\partial x^{2}} t_{11}^{(2)}+2 \frac{\partial^{2}}{\partial x \partial y} t_{12}^{(2)}+\frac{\partial^{2}}{\partial y^{2}} t_{22}^{(2)}=t
$$

We may iterate the process. We obtain, again formally, $\operatorname{grad} \circ \operatorname{grad} \circ \operatorname{grad} t^{(3)}$

$$
=\frac{\partial^{3}}{\partial x^{3}} t_{111}^{(3)}+3 \frac{\partial^{3}}{\partial x^{2} \partial y} t_{112}^{(3)}+3 \frac{\partial^{3}}{\partial x \partial y^{2}} t_{122}^{(3)}+\frac{\partial^{3}}{\partial y^{3}} t_{222}^{(3)}=t
$$


where, for example,

$$
t_{112}^{(3)}=t_{121}^{(3)}=t_{211}^{(3)}=\sum a_{n} e^{i n \cdot x}\left(\frac{i m^{2} n}{|n|^{6}}\right)
$$

Higher order formal integrals are easily constructed.

If we now replace partial differentiation by symmetric partial differentiation so that, for example, $\partial t_{1}^{(1)} / \partial x$ is approximated by

$$
\frac{t_{1}^{(1)}(x+h, y)-t_{1}^{(1)}(x-h, y)}{2 h}
$$

and $\partial^{3} t_{112}^{(3)} / \partial x^{2} \partial y$ is approximated by

$\frac{t_{112}^{(3)}(x+2 h, y+h)-t_{112}^{(3)}(x+2 h, y-h)+t_{112}^{(3)}(x-2 h, y+h)-t_{112}^{(3)}(x-2 h, y-h)-2 t_{112}^{(3)}(x, y+h)+2 t_{112}^{(3)}(x, y-h)}{(2 h)^{3}}$

then the left-hand sides of (4.34), (4.36), and (4.37) are approximated respectively by

$$
\begin{gathered}
\sum a_{n} e^{i n \cdot x}\left(\frac{m h \sin m h+n h \sin n h}{(m h)^{2}+(n h)^{2}}\right), \\
\sum a_{n} e^{i n \cdot x}\left(\frac{m h \sin m h+n h \sin n h}{(m h)^{2}+(n h)^{2}}\right)^{2},
\end{gathered}
$$

and

$$
\sum a_{n} e^{i n \cdot x}\left(\frac{m h \sin m h+n h \sin n h}{(m h)^{2}+(n h)^{2}}\right)^{3}
$$

For the symmetric approximation to the $p$ th derivative of the $p$ th formal integral we of course obtain

$$
\sum a_{n} e^{i n \cdot x}\left(\frac{m h \sin m h+n h \sin n h}{(m h)^{2}+(n h)^{2}}\right)^{p} .
$$

This motivates the following consistency theorem. (See also Lemma 4.2 and Theorem 4.4.)

THEOREM 4.5. Suppose $\sum_{m, n=0}^{\infty} a_{n} e^{i n \cdot x}=s$ and has bounded partial sums. Then if $p>2$,

$$
\lim _{h \rightarrow 0+} \sum_{m, n=0}^{\infty} a_{n} e^{i n \cdot x}\left(\frac{m h \sin m h+n h \sin n h}{(m h)^{2}+(n h)^{2}}\right)^{p}=s .
$$

(Interpret $(0 \sin 0+0 \sin 0) / 0^{2}$ to be 1.)

Proof. We apply Lemma 3.2 to

$$
\lambda_{m, n}(h)=\left(\frac{m h \sin m h+n h \sin n h}{(m h)^{2}+(n h)^{2}}\right)^{p}=f^{p}(m h, n h)
$$

where $f(u, v)=(u \sin u+v \sin v) /\left(u^{2}+v^{2}\right)$. Making use of inequalities of the type

$$
\left|\lambda_{m, n}(h)-\lambda_{m+1, n}(h)\right| \leqq \int_{m h}^{(m+1) h}\left|\frac{\partial}{\partial u} f^{p}(u, v)\right| d u,
$$


conditions (3.2a) are reduced to

$$
\begin{gathered}
\int_{0}^{\infty} \int_{0}^{\infty}\left|\frac{\partial^{2}}{\partial u \partial v} f^{p}(u, v)\right| d u d v \leqq C, \\
\int_{0}^{\infty}\left|\frac{\partial}{\partial u} f^{p}(u, v)\right| d u \leqq C, \\
\int_{0}^{\infty}\left|\frac{\partial}{\partial v} f^{p}(u, v)\right| d v \leqq C
\end{gathered}
$$

and

$$
\left|f^{p}(u, v)\right| \leqq C .
$$

To prove (4.45) change to polar coordinates and write

$$
\begin{aligned}
\int_{0}^{\infty} \int_{0}^{\infty}\left|\frac{\partial^{2}}{\partial u \partial v} f^{p}(u, v)\right| d u d v \\
=\int_{0}^{1} \int_{0}^{\pi / 2} \cdots r d r d \theta+\int_{1}^{\infty} \int_{0}^{\pi / 2} \cdots r d r d \theta=A+B
\end{aligned}
$$

Now

$$
\frac{\partial^{2}}{\partial u \partial v} f^{p}=p(p-1) f^{p-2} \frac{\partial f}{\partial u} \frac{\partial f}{\partial v}+p f^{p-1} \frac{\partial^{2} f}{\partial u \partial v}
$$

where

$$
\frac{\partial f}{\partial u}=\frac{r^{2}(\sin u+u \cos u)-2 u(u \sin u+v \sin v)}{r^{4}}
$$

and

$$
\frac{\partial^{2} f}{\partial u \partial v}=\frac{8 u v(u \sin u+v \sin v)-2 r^{2}[v \sin u+u \sin v+u v(\cos u+\cos v)]}{r^{6}}
$$

To estimate $A$ replace $\sin u$ by $u+O\left(r^{3}\right), \sin v$ by $v+O\left(r^{3}\right), \cos u$ and $\cos v$ by $1+O\left(r^{3}\right)$ to obtain that the numerators of (4.51) and (4.52) are $O\left(r^{4}\right)$ and $O\left(r^{6}\right)$ respectively as $r$ tends to zero. We handle $\partial f / \partial v$ symmetrically and observe that

$$
|f| \leqq 1
$$

These estimates show that the integrand in $A$ is $O(r)=O(1)$ as $r \rightarrow 0$ so that the integral is finite. To estimate $B$, use (4.51) and (4.52) to show that $\partial f / \partial u=O(1 / r)$, $\partial f / \partial v=O(1 / r)$ and $\partial^{2} f / \partial u \partial v=O\left(1 / r^{2}\right)$ at infinity. Since $f=O(1 / r)$ at infinity and the Jacobian is $r$, it follows that the integrand is $O\left(1 / r^{1+(p-2)}\right)$ at infinity and hence that $B$ is finite since $p>2$.

We now consider (4.46). Using $\partial f^{p} / \partial u=p f^{p-1} \partial f / \partial u$ and (4.53) we have

$$
\int_{0}^{\infty}\left|\frac{\partial}{\partial u} f^{p}\right| d u \leqq p \int_{0}^{1}\left|\frac{\partial f}{\partial u}\right| d u+p \int_{1}^{\infty}\left|f^{p-1} \frac{\partial f}{\partial u}\right| d u=p(A(v)+B(v))
$$


Again making third order estimates for sine and cosine we deduce from (4.51) that

$$
\left|\frac{\partial f}{\partial u}\right| \leqq \frac{2 u^{3}+2 u\left(u^{4}+v^{4}\right)}{\left(u^{2}+v^{2}\right)^{2}} \leqq \frac{2 u^{3}}{\left(u^{2}+v^{2}\right)^{2}}+2 u \leqq 4 u
$$

so that $A(v) \leqq 2$ for all values of $v$. Now let $u \geqq 1$. Since $r=\left(u^{2}+v^{2}\right)^{1 / 2} \geqq u \geqq 1$ and $r \geqq v$, it follows from (4.43) and (4.51) that

$$
|f| \leqq\left(\frac{u}{r}+\frac{v}{r}\right) \frac{1}{r} \leqq \frac{2}{r} \leqq \frac{2}{u}, \quad\left|f^{p-1}\right| \leqq \frac{2^{p-1}}{u^{p-1}}
$$

and

$$
\left|\frac{\partial f}{\partial u}\right| \leqq \frac{r^{2}(1+u)+2 u(u+v)}{r^{4}} \leqq \frac{6}{r} \leqq \frac{6}{u}
$$

so that $p>2$ implies

$$
B(v) \leqq \int_{1}^{\infty} \frac{12}{u^{p}} d u=\frac{12}{p-1}<12
$$

The validity of (4.46) with $C=14 p$ follows. The proof of (4.47) is symmetrical with the one just given. Also (4.48) is immediately clear from (4.53).

We must next verify conditions (3.2b) which in this case follow from

$$
\begin{gathered}
\int_{0}^{\infty} \int_{n h}^{(n+1) h}\left|\frac{\partial^{2}}{\partial x \partial y} f^{p}\right| d u d v \rightarrow 0 \text { as } h \rightarrow+0 \text { for each } n, \\
\int_{m h}^{(m+1) h} \int_{0}^{\infty}\left|\frac{\partial^{2}}{\partial x \partial y} f^{p}\right| d u d v \rightarrow 0 \text { as } h \rightarrow+0 \text { for each } m, \\
\left|\lambda_{m, n}(h)-\lambda_{m+1, n}(h)\right| \rightarrow 0 \text { as } h \rightarrow 0 \text { for all } m \text { and } n, \\
\left|\lambda_{m, n}(h)-\lambda_{m, n+1}(h)\right| \rightarrow 0 \text { as } h \rightarrow 0 \text { for all } m \text { and } n .
\end{gathered}
$$

For $n$ fixed and $\varepsilon>0$ given, we can choose $h$ small enough so that the integral in (4.59) is less than or equal to

$$
\int_{0}^{\infty} \int_{0}^{\varepsilon}\left|\frac{\partial^{2}}{\partial x \partial y} f^{p}\right| d u d v
$$

Using a simple argument involving the definition of improper integrals and the finiteness of (4.45), we see that (4.59) tends to zero with $\varepsilon$ and hence $h$.

The proof of (4.60) is symmetrical with that of (4.59). The verifications of (4.61) and (4.62) are routine. Finally, condition (3.2c) holds by the parenthetical remark following the statement of Theorem 4.5. This shows that the hypotheses of Lemma 3.2 are satisfied and completes the proof of Theorem 4.5. Observe that $p>2$ was necessary for the proof of (4.45) so that an approach to the theory of uniqueness via Theorem 4.5 would probably require at least three integrations. Actually, to avoid complications arising from terms with negative indices it might be better to use an even number of (hence at least four) integrations. 
V. On the almost everywhere summability of double Fourier series. Let $K_{n}^{\alpha}(t)$ be the $\alpha$ th Fejér means of the series $\frac{1}{2}+\cos t+\cos 2 t+\cdots$. We have

$$
D_{n}(t)=K_{n}^{\circ}(t)=\frac{1}{2}+\cos t+\cdots+\cos n t=\frac{\sin \left(n+\frac{1}{2}\right) t}{2 \sin (t / 2)}
$$

and, in general,

$$
K_{n}^{\alpha}(t)=\sum_{\nu=0}^{n} A_{n-\nu}^{\alpha-1} D_{v}(t) / A_{n}^{\alpha} \quad \text { where } A_{m}^{\beta}=\left(\begin{array}{c}
m+\beta \\
m
\end{array}\right)=\frac{(\beta+1)(\beta+2) \cdots(\beta+m)}{m !} .
$$

Consider the Fourier series $S[f]$ of a real-valued function $f(x, y)$ which is Lebesgue integrable over $T^{2}$. We say that $S[f]$ is $(C, \alpha, \beta)$ summable to $f$ at $(x, y)$ if

$$
\begin{aligned}
& \lim _{\min \{m, n\} \rightarrow \infty} \sigma_{m, n}^{\alpha, \beta}(f)(x, y) \\
& =\lim _{\min \{m, n\} \rightarrow \infty} \frac{1}{\pi^{2}} \int_{-\pi}^{\pi} \int_{-\pi}^{\pi} f(x-s, y-t) K_{m}^{\alpha}(s) K_{n}^{\beta}(t) d s d t=f(x, y) .
\end{aligned}
$$

In particular, $S[f]$ is $(C, \alpha, 0)$ summable to $f$ at $(x, y)$ if

$$
\begin{aligned}
& \lim _{\min \{m, n\} \rightarrow \infty} \sigma_{m, n}^{\alpha, 0}(f)(x, y) \\
& =\lim _{\min \{m, n\} \rightarrow \infty} \frac{1}{\pi^{2}} \int_{-\pi}^{\pi} \int_{-\pi}^{\pi} f(x-s, y-t) K_{m}^{\alpha}(s) D_{n}(t) d s d t=f(x, y) .
\end{aligned}
$$

In [24], A. Zygmund showed that if $f \in L^{p}\left(T^{2}\right), p>1$, and if $\alpha>0, \beta>0$, then $S[f]$ is $(C, \alpha, \beta)$ summable to $f(x, y)$ almost everywhere.

In [11], B. Jessen, J. Marcinkiewicz, and A. Zygmund generalized the result by weakening the hypothesis that $f \in L^{p}\left(T^{2}\right)$ to $f \in\left(L \log ^{+} L\right)\left(T^{2}\right)$, while still drawing the same conclusion.

A recent example of C. Fefferman [6] shows that the results of R. A. Hunt [9] and L. Carleson [1] concerning convergence of Fourier series of functions of one variable do not extend to unrestricted or even to restricted rectangular convergence for multiple Fourier series. (Fefferman [7], P. Sjolin [19], and N. Tevzadze [20] independently also proved that functions in $L^{p}\left(T^{k}\right)(p>1)$ have convergent Fourier series for square partial sums.) In other words, there exists a continuous $f \in L^{2}\left(T^{2}\right)$ for which $S[f]$ is not summable $(C, 0,0)$ to $f$ almost everywhere.

However, we can give the following theorem.

THEOREM 5.1. If, for almost every $x$ in $T^{1}, f(x, y) \in L\left(\log ^{+} L\right)^{2}\left(T^{1}(y)\right)$ and if $g(x)=\int_{T^{1}}|f(x, y)|\left(\log ^{+}|f(x, y)|\right)^{2} d y \in L\left(T^{1}(x)\right)$, then, for every positive $\alpha, f$ is summable $(C, \alpha, 0)$ almost everywhere. In particular, if $f \in L^{p}\left(T^{2}\right), p>1$, the conclusion holds.

THEOREM 5.2. Let $\alpha>0$. Under the hypotheses of the first part of Theorem 5.1 we have

$$
\int_{-\pi}^{\pi} \int_{-\pi}^{\pi}\left|\sigma_{*}^{\alpha, 0}(x, y)\right| d x d y \leqq A_{\alpha} \int_{-\pi}^{\pi} \int_{-\pi}^{\pi}|f|\left(\log ^{+}|f|\right)^{2} d x d y+A_{\alpha}
$$


and if $f \in L^{p}\left(T^{2}\right), p>1$, then

$$
\int_{-\pi}^{\pi} \int_{-\pi}^{\pi}\left|\sigma_{*}^{\alpha, 0}(x, y)\right|^{p} d x d y \leqq C_{\alpha, p} \int_{-\pi}^{\pi} \int_{-\pi}^{\pi}|f(x, y)|^{p} d x d y
$$

where

$$
\sigma_{*}^{\alpha, 0}(x, y)=\lim _{\min \{m, n\} \rightarrow \infty}\left|\sigma_{m, n}^{\alpha, 0}(x, y)\right|
$$

We begin by proving Theorem 5.2. We will only prove (5.3) since the proof of (5.4) is similar. Without loss of generality let $0<\alpha<1$. Since $\left|K_{m}^{\alpha}(x)\right|$ satisfies the conditions

$$
\begin{gathered}
1 \leqq \frac{1}{\pi} \int_{-\pi}^{\pi}\left|K_{m}^{\alpha}(x)\right| d x=C_{m}(\alpha) \leqq C_{\alpha} \text { for all } m, \\
\max _{\delta \leqq|x| \leqq \pi}\left|K_{n}^{\alpha}(x)\right| \rightarrow 0 \text { as } n \rightarrow \infty \text { for each } \delta,
\end{gathered}
$$

and

$$
\left|K_{n}^{\alpha}(x)\right| \leqq \min \left\{2 n, A_{\alpha} n^{-\alpha}|x|^{-(\alpha+1)}\right\},
$$

it follows that if $h(x) \in L^{1}\left(T^{1}\right)$, then

$$
\frac{1}{\pi C_{m}(\alpha)} \int_{-\pi}^{\pi}\left|K_{m}^{\alpha}(t)\right| h(x-t) d t \rightarrow h(x)
$$

as $m \rightarrow \infty$ for almost every $x$ [23, Vol. I, p. 94]. From (5.6) and (5.9) we deduce that for $h \in L^{1}\left(T^{1}\right)$

$$
\sigma_{* *}^{\alpha}(h)(x)=\limsup _{n \rightarrow \infty} \frac{1}{\pi} \int_{-\pi}^{\pi}\left|K_{n}^{\alpha}(t)\right||h(x-t)| d t \leqq C_{\alpha}|h(x)|
$$

for almost every $x$.

Let

$$
M_{2} f(x, y)=\sup _{n}\left|\frac{1}{\pi} \int_{-\pi}^{\pi} D_{n}(t) f(x, y-t) d t\right|
$$

For almost every $x, f(x, y) \in L\left(\log ^{+} L\right)^{2}$ as a function of $y$. For such $x$ 's we have

$$
\int_{-\pi}^{\pi} M_{2} f(x, y) d y \leqq A \int_{-\pi}^{\pi}|f(x, y)|\left(\log ^{+}|f(x, y)|\right)^{2} d y+A .
$$

(See R. Hunt [9, p. 235].) Integrating (5.11) with respect to $x$ we find that $M_{2} \in L^{1}\left(T^{2}\right)$ and

$$
\int_{-\pi}^{\pi} \int_{-\pi}^{\pi} M_{2} f(x, y) d y d x \leqq A \int_{-\pi}^{\pi} \int_{-\pi}^{\pi}|f|\left(\log ^{+}|f|\right)^{2} d y d x+2 \pi A .
$$


Now fix a $y$ for which $M_{2} f(x, y)$ is integrable as a function of $x$. For almost every $x$ we have by (5.10) and the definition of $M_{2}$,

$$
\begin{aligned}
\sigma_{*}^{\alpha, 0}(f)(x, y) & =\limsup _{\min \{m, n\} \rightarrow \infty}\left|\frac{1}{\pi} \int_{-\pi}^{\pi} K_{m}^{\alpha}(s)\left\{\frac{1}{\pi} \int_{-\pi}^{\pi} D_{n}(t) f(x-s, y-t) d t\right\} d s\right| \\
& \leqq \limsup _{m \rightarrow \infty} \frac{1}{\pi} \int_{-n}^{\pi}\left|K_{m}^{\alpha}(s)\right| M_{2} f(x-s, y) d s \leqq C_{\alpha} M_{2} f(x, y) .
\end{aligned}
$$

Since (5.13) holds almost everywhere, combining (5.12) and (5.13) proves (5.3).

The argument that proves Theorem 5.1 from Theorem 5.2 is classical. We reproduce the proof here for completeness. (See [11, p. 221].) First apply (5.3) to the function $\lambda f$ where $\lambda>0$ is a constant obtaining

$$
\int_{-\pi}^{\pi} \int_{-\pi}^{\pi} \sigma_{*}^{\alpha, 0}(f)(x, y) d x d y \leq A_{\alpha} \int_{-\pi}^{\pi} \int_{-\pi}^{\pi}|f|\left(\log ^{+}|\lambda f|\right)^{2} d x d y+\frac{A_{\alpha}}{\lambda} .
$$

Let $\varepsilon>0$ be given. Choose $\lambda$ so large that

$$
A_{\alpha} / \lambda<\varepsilon^{2} / 8
$$

Decompose $f, f=f^{\prime}+f^{\prime \prime}$ where $f^{\prime}$ is a trigonometric polynomial,

$$
\int_{-\pi}^{\pi} \int_{-\pi}^{\pi}\left|f^{\prime \prime}\right| d x d y<\frac{\varepsilon^{2}}{4}
$$

and

$$
A_{\alpha} \int_{-\pi}^{\pi} \int_{-\pi}^{\pi}\left|f^{\prime \prime}\right|\left(\log +\lambda\left|f^{\prime \prime}\right|\right)^{2} d x d y<\frac{\varepsilon^{2}}{8}
$$

(See [23, Vol. II, p. 304].) From (5.16) and Tchebycheff's inequality

$$
\left|\left\{(x, y)|| f^{\prime \prime}(x, y) \mid>\varepsilon / 2\right\}\right|<\varepsilon / 2 .
$$

From (5.15), (5.17), (5.14) applied to $f^{\prime \prime}$, and Tchebycheff's inequality we have

$$
\left|\left\{(x, y) \mid \sigma_{*}^{\alpha, 0}\left(f^{\prime \prime}\right)(x, y)>\varepsilon / 2\right\}\right|<\varepsilon / 2 .
$$

From (5.18) and (5.19) we see that if $E(\varepsilon)$ is the set of all $(x, y)$ for which either $\left|f^{\prime \prime}\right|>\varepsilon / 2$ or $\sigma_{*}^{\alpha, 0}\left(f^{\prime \prime}\right)>\varepsilon / 2$, then $E(\varepsilon)$ has measure $<\varepsilon$. Since

$$
\sigma_{m, n}(f)-f=\sigma_{m, n}\left(f^{\prime}\right)-f^{\prime}+\sigma_{m, n}\left(f^{\prime \prime}\right)-f^{\prime \prime},
$$

we see that, outside the set $E(\varepsilon)$,

$$
\lim _{\min \{m, n\} \rightarrow \infty}\left|\sigma_{m, n}(f)-f\right| \leqq \sigma_{*}^{\alpha, 0}\left(f^{\prime \prime}\right)+\left|f^{\prime \prime}\right|<\frac{\varepsilon}{2}+\frac{\varepsilon}{2}=\varepsilon .
$$

Now if $\sigma_{m, n}$ did not converge almost everywhere, it would fail to converge on a set $F,|F|>0$. Write

$$
F=\bigcup_{n=1}^{\infty} F_{n}=\bigcup_{n=1}^{\infty}\left\{(x, y)\left|\lim _{\min \{m, n\} \rightarrow \infty}\right| \sigma_{m, n}(f)-f \mid>\frac{1}{n}\right\} .
$$


Some $F_{n}$ would have positive measure, say $\left|F_{n_{0}}\right|>\delta>0$. Picking $\varepsilon<\min \left\{1 / n_{0}, \delta\right\}$ and applying (5.20) would yield a contradiction, since $F_{n_{0}} \subset E(\varepsilon)$ but $\left|F_{n_{0}}\right|>\delta>\varepsilon$ $\geqq|E(\varepsilon)|$. Hence, $\sigma_{m, n}$ does converge almost everywhere. This proves Theorem 5.1.

The space $L\left(\log ^{+} L\right)^{2}$ is not the largest possible space that might be used in the statements of Theorems 5.1 and 5.2. Sjolin [19] has proved that the hypothesis for the convergence of the Fourier series in one variable may be weakened from " $f(x)$ belongs to $L\left(\log ^{+} L\right)^{2}$ on $T^{1}$ " to " $f(x)$ belongs to $L\left(\log ^{+} L\right)\left(\log ^{+} \log ^{+} L\right)$ on $T^{1}$ ". It is clear that as further results are obtained in the theory of functions of one variable, one may correspondingly weaken the hypotheses in the first parts of Theorems 5.1 and 5.2 while still obtaining the same conclusions.

\section{BIBLIOGRAPHY}

1. L. Carleson, On convergence and growth of partial sums of Fourier series, Acta Math. 116 (1966), 135-157. MR 33 \#7774.

2. P. J. Cohen, Topics in the theory of uniqueness of trigonometrical series, Thesis, University of Chicago, Chicago, Ill., 1958.

3. R. Cooke, A Cantor-Lebesgue theorem in two dimensions, Proc. Amer. Math. Soc. 30 (1971), 547-550.

4. G. Cross, Multiple trigonometric series of a particular type, Duke Math. J. 29 (1962), 489-495. MR 25 \#4304.

5. F. Elgar, Mondrian, Frederick A. Praeger, New York, 1968.

6. C. Fefferman, On the divergence of multiple Fourier series, Bull. Amer. Math. Soc. 77 (1971), 191-195.

7. - On the convergence of multiple Fourier series, Bull. Amer. Math. Soc. 77 (1971), 744-745.

8. H. Geiringer, Trigonometrische Doppelreihen, Monatsh. Math. Phys. 29 (1918), 65-144.

9. R. A. Hunt, On the convergence of Fourier series, Proc. Conf. Orthogonal Expansions and their Continuous Analogues (Edwardsville, Ill., 1967), Southern Illinois Univ. Press, Carbondale, Ill., 1968, pp. 235-255. MR 38 \#6296.

10. S. Igari, Lectures on Fourier series of several variables, University of Wisconsin Lecture Notes, Madison, Wis., 1968, pp. 1-208.

11. B. Jessen, J. Marcinkiewicz and A. Zygmund, Note on the differentiability of multiple integrals, Fund. Math. 25 (1935), 217-234.

12. M. H. Nasibov, On the uniqueness of the expansion of a function of two variables into trigonometric series, Izv. Akad. Nauk Azerbaĭdžan SSR Ser. Fiz.-Tehn. Mat. Nauk 1964, no. 2, 13-22. MR 30 \#1349.

13. H. Rademacher, Lectures on elementary number theory, Pure and Appl. Sciences, Blaisdell, New York, 1964. MR 30 \#1079.

14. G. E. Reves and O. Szász, Some theorems on double trigonometric series, Duke Math. J. 9 (1942), 693-705. MR 4, 217.

15. G. M. Robison, Divergent double sequences and series, Trans. Amer. Math. Soc. 28 (1926), 50-73.

16. V. L. Shapiro, Uniqueness of multiple trigonometric series, Ann. of Math. (2) 66 (1957), 467-480. MR 19, 854; 1432.

17. - The approximate divergence operator, Proc. Amer. Math. Soc. 20 (1969), 55-60. MR 38 \#4918.

18. — Fourier series in several variables, Bull. Amer. Math. Soc. 70 (1964), 48-93. MR 28 \#1448. 
19. P. Sjolin, On the convergence almost everywhere of certain singular integrals and multiple Fourier series, Ark. Mat. 9 (1971), 65-90.

20. N. Tevzadze, On the convergence of double Fourier series of quadratic summable functions, Soobšč. Akad. Nauk Gruzin. SSR 5 (1970), 277-279.

21. E. C. Titchmarsh, The theory of functions, 2nd ed., Oxford Univ. Press, Oxford, 1939.

22. I. E. Zak, On Riemann summability of double numerical series, Soobšč. Akad. Nauk Gruzin. SSR 13 (1952), 587-593. MR 14, 1079.

23. A. Zygmund, Trigonometric series. Vols. 1, 2, 2nd rev. ed., Cambridge Univ. Press, New York, 1959. MR 21 \#6498.

24. - On the differentiability of multiple integrals, Fund. Math. 23 (1934), 143-149.

25. - Oral communication concerning spherical convergence.

DePaul University, Chicago, Illinois 60614

Current address (Welland): University of Missouri-St. Louis, St. Louis, Missouri 63121 